\title{
LOCALIZED AND EXPANDING ENTIRE SOLUTIONS OF REACTION-DIFFUSION EQUATIONS
}

\author{
F. HAMEL AND H. NINOMIYA
}

\begin{abstract}
This paper is concerned with the spatio-temporal dynamics of nonnegative bounded entire solutions of some reaction-diffusion equations in $\mathbb{R}^{N}$ in any space dimension $N$. The solutions are assumed to be localized in the past. Under certain conditions on the reaction term, the solutions are then proved to be time-independent or heteroclinic connections between different steady states. Furthermore, either they are localized uniformly in time, or they converge to a constant steady state and spread at large time. This result is then applied to some specific bistable-type reactions.
\end{abstract}

In memory of Geneviève Raugel, with admiration and respect

Keywords: Reaction-diffusion equations, entire solutions, extinction, propagation

\section{InTRODUCTION AND THE MAIN RESULT}

In this paper we are concerned with nonnegative bounded entire solutions of the following reaction-diffusion equation:

$$
u_{t}=\Delta u+f(u), \quad t \in \mathbb{R}, \quad x \in \mathbb{R}^{N},
$$

where $f:[0,+\infty) \rightarrow \mathbb{R}$ is a $C^{1}$ function such that

$$
f(0)=0 \text { and } f^{\prime}(0)<0 .
$$

The solutions are always understood in the classical sense $C_{t, x}^{1,2}\left(\mathbb{R} \times \mathbb{R}^{N}\right)$, from the parabolic regularity theory. Notice immediately that, for a nonnegative bounded solution $u$ of (1.1), either $u(t, x)=0$ for all $(t, x) \in \mathbb{R} \times \mathbb{R}^{N}$, or $u(t, x)>0$ for all $(t, x) \in \mathbb{R} \times \mathbb{R}^{N}$, from the strong parabolic maximum principle and the uniqueness of the bounded solutions for the associated Cauchy problem.

The solutions $u$ are called entire as they are defined for all $t \in \mathbb{R}$ and $x \in \mathbb{R}^{N}$. The solution $u$ of (1.1) is called bounded if $u$ is an entire solution of (1.1) and

$$
\sup _{(t, x) \in \mathbb{R}^{N+1}}|u(t, x)|<\infty .
$$

We are especially interested in the description of their limit profiles as $t \rightarrow \pm \infty$. If a solution $u$ converges, in some sense to be made precise, to some limit states $\phi_{ \pm}$as $t \rightarrow \pm \infty$, then $u$ is a heteroclinic connection between $\phi_{-}$and $\phi_{+}$if $\phi_{-} \neq \phi_{+}$, while it is homoclinic to $\phi_{ \pm}$if $\phi_{-}=\phi_{+}$(we will actually prove that the homoclinic connections reduce to timeindependent solutions under the assumptions in this paper). The description and the properties of the entire solutions of (1.1) are of particular importance such as, for any element $\varphi$ of the $\omega$-limit set of any nonnegative initial condition of the associated Cauchy problem giving 
rise to a bounded global solution, and for any $t_{0} \in \mathbb{R}$, there is a bounded entire solution $u$ of (1.1) such that $u\left(t_{0}, \cdot\right)=\varphi$.

1.1. Localized solutions in the past and localized steady states. We are interested in solutions that are localized in the past, in the sense that

$$
u(t, x) \rightarrow 0 \text { as }|x| \rightarrow+\infty \text {, uniformly in } t \leq 0 .
$$

Throughout the paper, $x \mapsto|x|$ denotes the Euclidean norm in $\mathbb{R}^{N},(x, y) \mapsto x \cdot y$ denotes the Euclidean inner product, $B(x, R)$ denotes the open Euclidean ball of center $x \in \mathbb{R}^{N}$ and radius $R>0$, and $B_{R}=B(0, R)$. Notice that the condition $f(0)=0$ is then forced by (1.3). Furthermore, from standard parabolic estimates and the boundedness of $u$, condition (1.3) is equivalent to $\lim _{|x| \rightarrow+\infty} u(t, x)=0$ uniformly in $t \leq t_{0}$ for some (or equivalently for all) $t_{0} \in \mathbb{R}$. This, however, does not necessarily mean that $\lim _{|x| \rightarrow+\infty} u(t, x)=0$ uniformly in $t \in \mathbb{R}$ (such solutions are called uniformly localized), and one of the main features of the paper is to show a dichotomy between the solutions that are uniformly localized and those that spread as $t \rightarrow+\infty$.

The description of the positive bounded solutions of (1.1) satisfying (1.3) is closely related to the study of the positive bounded localized steady states $\phi \in C^{2}\left(\mathbb{R}^{N}\right)$, solving

$$
\left\{\begin{array}{l}
\Delta \phi+f(\phi)=0 \text { and } \phi>0 \text { in } \mathbb{R}^{N} \\
\phi(x) \rightarrow 0 \text { as }|x| \rightarrow+\infty
\end{array}\right.
$$

Under the condition $f^{\prime}(0)<0$, it is known [27, 38, 39] that any solution $\phi$ of (1.4) is radially symmetric and decreasing with respect to its center, namely there exist a point $x_{0} \in \mathbb{R}^{N}$ and a $C^{2}([0,+\infty))$ function $\Phi$ such that $\Phi^{\prime}<0$ in $(0,+\infty)$ and

$$
\phi(x)=\Phi\left(\left|x-x_{0}\right|\right) \text { for all } x \in \mathbb{R}^{N} .
$$

It then follows from the strong maximum principle applied to $\phi$ that

$$
f(\Phi(0))=f\left(\max _{\mathbb{R}^{N}} \phi\right)>0
$$

hence, together with (1.2), there is a unique real number $m_{\phi}$ such that

$$
0<m_{\phi}<\max _{\mathbb{R}^{N}} \phi, \quad f\left(m_{\phi}\right)=0 \text { and } f>0 \text { in }\left(m_{\phi}, \max _{\mathbb{R}^{N}} \phi\right] .
$$

Lastly, since

$$
\Phi^{\prime \prime}(r)+\frac{N-1}{r} \Phi^{\prime}(r)+f(\Phi(r))=0 \text { for all } r \in(0,+\infty)
$$

and $\Phi^{\prime}(0)=\Phi^{\prime}(+\infty)=0$ (the limit $\Phi^{\prime}(+\infty)=0$ coming from (1.4)-(1.5) and standard elliptic estimates), integrating the above equation against $\Phi^{\prime}$ over $(0,+\infty)$ yields $F\left(\max _{\mathbb{R}^{N}} \phi\right)=F(\Phi(0))=0$ if $N=1$ and $F\left(\max _{\mathbb{R}^{N}} \phi\right)=F(\Phi(0))>0$ if $N \geq 2$, where

$$
F(s)=\int_{0}^{s} f(\sigma) d \sigma \text { for } s \geq 0 .
$$


Since $f\left(\max _{\mathbb{R}^{N}} \phi\right)>0$, there is then $\eta>0$ such that $f>0$ in $\left[\max _{\mathbb{R}^{N}} \phi-\eta, \max _{\mathbb{R}^{N}} \phi+\eta\right]$ and $F>0$ in $\left(\max _{\mathbb{R}^{N}} \phi, \max _{\mathbb{R}^{N}} \phi+\eta\right]$ if $N=1\left(\right.$ resp. in $\left[\max _{\mathbb{R}^{N}} \phi, \max _{\mathbb{R}^{N}} \phi+\eta\right]$ if $\left.N \geq 2\right)$. In the sequel, we also set

$$
M_{\phi}=\inf \left\{s \geq \max _{\mathbb{R}^{N}} \phi: f(s)=0\right\} \in\left(\max _{\mathbb{R}^{N}} \phi,+\infty\right] .
$$

Notice that

$$
m_{\phi}<\max _{\mathbb{R}^{N}} \phi<M_{\phi} \text { and } f>0 \text { in }\left(m_{\phi}, M_{\phi}\right),
$$

and that $M_{\phi}$ may be equal to $+\infty$ (we refer to some specific examples in Section 2).

Furthermore, not only the steady states of (1.4) are radially symmetric and decreasing with respect to some center, but so are the bounded entire solutions of (1.1) which are localized in the past. Namely, it follows from [54] that, for any positive bounded solution $u$ of (1.1)-(1.3), there is a point $x_{0} \in \mathbb{R}^{N}$ such that

$$
\begin{cases}u(t, x)=u(t, y) & \text { for all }(t, x, y) \in \mathbb{R} \times \mathbb{R}^{N} \times \mathbb{R}^{N} \text { with }\left|x-x_{0}\right|=\left|y-x_{0}\right|, \\ \nabla u(t, x) \cdot\left(x-x_{0}\right)<0 & \text { for all }(t, x) \in \mathbb{R} \times \mathbb{R}^{N} \text { with } x \neq x_{0} .\end{cases}
$$

1.2. The main result. In the following theorem, which is the main result of the paper, we call $\mathcal{E}$ the set of $C^{2}\left(\mathbb{R}^{N}\right)$ solutions of (1.4) and, for any continuous bounded function $\varphi: \mathbb{R}^{N} \rightarrow \mathbb{R}$ and any set $\mathcal{A}$ of continuous bounded functions, we denote

$$
\operatorname{dist}(\varphi, \mathcal{A})=\inf _{\psi \in \mathcal{A}}\|\varphi-\psi\|_{L^{\infty}\left(\mathbb{R}^{N}\right)}
$$

Theorem 1.1. Assume that $f$ satisfies (1.2) and

$$
F<0 \text { in }\left(0, m_{\phi}\right] \text { for all } \phi \in \mathcal{E} \text {. }
$$

If there exists a positive bounded solution $u$ of (1.1) satisfying (1.3), then $\mathcal{E} \neq \emptyset$ and

$$
\operatorname{dist}(u(t, \cdot), \mathcal{E}) \rightarrow 0 \text { as } t \rightarrow-\infty \text {. }
$$

Furthermore,

(i) either $u(t, \cdot) \rightarrow 0$ uniformly in $\mathbb{R}^{N}$ as $t \rightarrow+\infty$,

(ii) or there is $\phi \in \mathcal{E}$ such that $u(t, \cdot) \rightarrow \phi$ uniformly in $\mathbb{R}^{N}$ as $t \rightarrow+\infty$,

(iii) or else there is a continuous function $\xi: \mathbb{R} \rightarrow \mathbb{R}$ depending on $u$ and some positive constants $M$ and $c$ only depending on $f$ such that

$$
\left\{\begin{array}{l}
\limsup _{t \rightarrow+\infty}\left(\max _{|x| \leq \xi(t)-A}|u(t, x)-M|\right) \rightarrow 0 \\
\limsup _{t \rightarrow+\infty}\left(\max _{|x| \geq \xi(t)+A} u(t, x)\right) \rightarrow 0
\end{array} \text { as } A \rightarrow+\infty\right.
$$

and

$$
\lim _{t \rightarrow+\infty} \frac{\xi(t)}{t}=c
$$

with $f(M)=0, f^{\prime}(M) \leq 0$ and $c$ characterized by the existence of a function $\varphi \in C^{2}(\mathbb{R})$ solving

$$
\varphi^{\prime \prime}+c \varphi^{\prime}+f(\varphi)=0 \text { in } \mathbb{R}, \quad \varphi^{\prime}<0 \text { in } \mathbb{R}, \quad \varphi(-\infty)=M, \quad \varphi(+\infty)=0 .
$$


Property (1.12) means that the $\alpha$-limit sets, with respect to the uniform convergence in $\mathbb{R}^{N}$, of the positive bounded solutions $u$ of (1.1) consist of steady states solving (1.4). As far as the behavior of a solution $u$ as $t \rightarrow+\infty$ is concerned, it turns out that, in both cases (i) and (ii), $u(t, x) \rightarrow 0$ as $|x| \rightarrow+\infty$ uniformly in $t \in \mathbb{R}$, namely $u$ is then called uniformly localized. As a consequence, the conclusion of Theorem 1.1 means there is a dichotomy between the uniformly localized solutions and the ones which converge locally uniformly to a positive constant, with a positive spreading rate. Notice that in all cases (i), (ii) and (iii), the solution $u$ converges locally uniformly in $\mathbb{R}^{N}$ as $t \rightarrow+\infty$ to a steady state (either a necessarily non-constant solution of (1.4), or the constants 0 or $M$ ) and its $\omega$-limit set (with respect to the uniform convergence in cases (i) and (ii) and to the locally uniform convergence in case (iii)) is a singleton.

This situation is in contrast with some non-convergence and even non-quasiconvergence results of some positive bounded solutions of the Cauchy problems of the Fujita equation

$$
u_{t}=\Delta u+u^{p}
$$

for which the $\omega$-limit set (with respect to the locally uniform convergence) of some initial conditions may not be reduced to a single steady state (non-convergence) or may even contain other elements than steady states (non-quasiconvergence). Such results have been proved in [57, 60, 61] for (1.16) in high dimensions $N$ for some ranges of values of $p$, even for solutions which are localized at large time (see also [18, 55, 56] for further non-quasiconvergence results with non-localized oscillating initial conditions and bistable nonlinearities of the type (1.20) below). On the other hand, convergence or quasiconvergence hold for all functions $f$ in dimension $N=1$ with compactly supported initial conditions [14] or for generic functions $f$ in any dimension $N \geq 1$ with initial conditions converging to 0 at infinity [42, 43], while the existence of at least one steady state in bounded trajectories has been shown in dimensions $N \leq 2$ [25]. We refer to [47, 48] for further convergence or quasiconvergence results for some bistable, ignition or monostable nonlinearities $f$ in any dimension $N \geq 1$ with radially decreasing initial conditions, and to [32] for a general overview on convergence results for gradient-like parabolic or hyperbolic equations.

Remark 1.2. It actually follows from the proof of Theorem 1.1, in particular from Steps 4 and 5 in Section 4.2, that a similar result as (1.13)-(1.14) holds for the spreading solutions of the associated Cauchy problem with localized initial conditions. More precisely, let $0<$ $m<M$ be given, and let $f:[0, M] \rightarrow \mathbb{R}$ be a given $C^{1}([0, M])$ function such that $f(0)=$ $f(m)=f(M)=0, f^{\prime}(0)<0, f>0$ in $(m, M), F<0$ in $(0, m]$ and $F(M)>0$. Let $u_{0}: \mathbb{R}^{N} \rightarrow[0, M]$ be a continuous function such that $\lim _{|x| \rightarrow+\infty} u_{0}(x)=0$ and $u_{0} \not \equiv 0$ in $\mathbb{R}^{N}$. Now, if the bounded solution $u$ of the Cauchy problem associated with (1.1) with initial condition $u_{0}$ is assumed to be such that

$$
u(t, \cdot) \rightarrow M \text { as } t \rightarrow+\infty \text { locally uniformly in } \mathbb{R}^{N},
$$

then properties (1.13)-(1.14) still hold for some continuous function $\xi:[0,+\infty) \rightarrow \mathbb{R}$, where $c$ is characterized by the existence of a solution $\varphi \in C^{2}(\mathbb{R})$ of (1.15). Notice that property (1.13) implies in particular that, for each $0<\varepsilon \leq M / 2$ and each unit vector $e$, the diameter of the set $\{r \geq 0: \varepsilon \leq u(t, r e) \leq M-\varepsilon\}$ is bounded as $t \rightarrow+\infty$ (see also 
the second paragraph after Remark 1.3). Furthermore, the same conclusion holds if, instead of $\lim _{|x| \rightarrow+\infty} u_{0}(x)=0$, one assumes that $\limsup _{|x| \rightarrow+\infty} u_{0}(x) \leq \eta$, with $\eta>0$ such that $f<0$ in $(0, \eta]$ (in that case, one has $\lim \sup _{|x| \rightarrow+\infty} u(t, x) \rightarrow 0$ as $t \rightarrow+\infty$ ).

1.3. Comments about the assumptions (1.2) and (1.11) on $f$, and (1.3) on $u$. Let us make in the following paragraphs some comments about the role and necessity of the various assumptions on $f$ and $u$ used in Theorem 1.1.

Let us first discuss the linear stability assumption (1.2) on $f$. Firstly, as already emphasized, the equality $f(0)=0$ is necessary for (1.3) to hold. Secondly, if $f^{\prime}(0)=0$, then Theorem 1.1 does not hold in general. For instance, in dimensions $N \geq 3$ and for $(N+2) /(N-2)<p<p_{L}$ with $p_{L}=(N-4) /(N-10)$ if $N \geq 11$ and $p_{L}=\infty$ if $N \leq 10$, the Fujita equation (1.16) admits positive bounded entire solutions $u$ which are uniformly localized and are homoclinic to 0 , in the sense that $\|u(t, \cdot)\|_{L^{\infty}\left(\mathbb{R}^{N}\right)} \rightarrow 0$ as $t \rightarrow \pm \infty$, see [21]. Thirdly, if $f^{\prime}(0)>0$, then Theorem 1.1 does not make sense in general. Consider for instance a $C^{2}$ concave function $f:[0,+\infty) \rightarrow \mathbb{R}$ such that $f(0)=0, f^{\prime}(0)>0$ and $f(b)=0$ for some $b>0$ (then, $f(s)<0$ for all $s>b$ ). Any nonnegative bounded entire solution $u$ of (1.1) and (1.3) necessarily satisfies $0 \leq u<b$ in $\mathbb{R} \times \mathbb{R}^{N}$ from the maximum principle. Furthermore, $\max _{\mathbb{R}^{N}} u(t, \cdot)<b$ for all $t$ negative enough (and then for all $t \in \mathbb{R}$ ), and it then follows from [33] that $u(t, x)$ is a function of $t$ alone and (1.3) then yields $u \equiv 0$ in $\mathbb{R} \times \mathbb{R}^{N}$.

Let us now focus on condition (1.11) on $f$. Notice first that it implies that the map $\phi \mapsto m_{\phi}$ is constant in $\mathcal{E}$. Indeed, for any $\phi, \phi^{\prime} \in \mathcal{E}$, one has $F<0$ in $\left(0, m_{\phi^{\prime}}\right]$ by (1.11), while $F\left(\max _{\mathbb{R}^{N}} \phi\right) \geq 0$ and $f\left(\max _{\mathbb{R}^{N}} \phi\right)>0$. Hence, owing to the definition (1.7) of $m_{\phi}$ and the fact that $f\left(m_{\phi^{\prime}}\right)=0$, one infers that $m_{\phi^{\prime}} \leq m_{\phi}$. Otherwise $m_{\phi}<\max _{\mathbb{R}^{N}} \phi<m_{\phi^{\prime}}$ and thus $F\left(\max _{\mathbb{R}^{N}} \phi\right)<0$, which is a contradiction. Finally $m_{\phi}=m_{\phi^{\prime}}$ since $\phi$ and $\phi^{\prime}$ are arbitrary in $\mathcal{E}$. As a consequence, one also gets that the function $\phi \mapsto M_{\phi}$ is constant in $\mathcal{E}$, where the quantity $M_{\phi}$ has been defined in (1.9).

Remark 1.3. From the proof of Theorem 1.1, it turns out that, in case (iii) of Theorem 1.1, one necessarily has $M_{\phi}<+\infty$ for any (and all) $\phi \in \mathcal{E}$ and

$$
M=M_{\phi} \text {. }
$$

In particular, if the solution $u$ spreads, it can not converge to an intermediate state smaller than $M$ and the limit state $M$ does not depend on the solution $u$ itself. As a matter of fact, since $F<0$ in $\left(0, m_{\phi}\right], m_{\phi}<\max _{\mathbb{R}^{N}} \phi<M_{\phi}, F\left(\max _{\mathbb{R}^{N}} \phi\right) \geq 0, f>0$ in $\left(m_{\phi}, M_{\phi}\right)$ and $f\left(M_{\phi}\right)=0$ for all $\phi \in \mathcal{E}$, one then infers that $M=M_{\phi}$ is the smallest zero of $f$ for which $F(M)>0$, that is,

$$
M=\min \{s \geq 0: f(s)=0 \text { and } F(s)>0\} .
$$

Therefore, $M$ only depends on the function $f$. Notice that this also implies that case (iii) is ruled out if $f>0$ in $\left(m_{\phi},+\infty\right)$ for any (and all) $\phi \in \mathcal{E}$, hence only cases (i) or (ii) may occur in this case.

Together with (1.2), assumption (1.11) plays a key-role in the dichotomy results between the uniformly localized solutions and the solutions converging as $t \rightarrow+\infty$ to a positive constant with a positive spreading rate. Without the assumption (1.11), the solutions $u$ of (1.1) may well converge locally uniformly in $\mathbb{R}^{N}$ as $t \rightarrow+\infty$ to a steady state $\phi$ such 
that $\lim _{|x| \rightarrow+\infty} \phi(x)>0$ (such behaviors are known for the solutions of the associated Cauchy problem with localized initial conditions in dimension $N=1$ [42, 43] or with compactly supported initial conditions in dimensions $N \geq 1$ [16]).

The assumption (1.11) is also essential in the proof of formula (1.13) saying that, for spreading solutions, the transition between $M-\varepsilon$ and $\varepsilon$ (for any $0<\varepsilon \leq M / 2$ ) has bounded width in any radial direction as $t \rightarrow+\infty$. This property refers to the notion of transition fronts (here, as $t \rightarrow+\infty$ ) introduced in [5]. In other words, the assumption (1.11) prevents the existence of terraces made of stacked propagating fronts between the top value $M$ and the zero state. The existence and attractivity of radial terraces with radial positions $(0<) \xi_{1}(t)<$ $\cdots<\xi_{m}(t)$ has been proved in [15] under an additional non-degeneracy assumption of all zeroes of $f$ in $[0, M]$ (see also [17, 20, 28, 43] for further results on terraces for homogeneous or spatially periodic equations in dimension $N=1,62,63$ for the existence of one-dimensional and radially symmetric terraces for gradient multistable systems, [58, 59] for the existence of planar terraces for solutions with front-like initial data in $\mathbb{R}^{N}$, and [29] for the existence of terraces in spatially periodic equations in $\left.\mathbb{R}^{N}\right)$. The limit values of the ratios of $\xi_{i}(t) / t$ are also explicit, see [15]. Here, especially thanks to (1.11), only a single radial layer can exist, and the asymptotic position $\xi(t)$ of that layer at large times is given in terms of the unique speed of a traveling front $\varphi$ connecting 0 and $M$ for problem (1.15). We point out that $M$ is asymptotically stable from below since $f$ is positive in a left neighborhood of $M$, but $M$ may not be linearly stable, in the sense that $f^{\prime}(M)$ may vanish. Actually, formula (1.14) is proved even if $f^{\prime}(M)=0$ (notice in particular that $f$ may not be monotone in a left neighborhood of $M)^{1}$ and, as such, up to our knowledge, the spreading properties (1.13)-(1.14) are new even in dimension $N=1$. The exact position of the layer $\xi(t)$ and a quantitative estimate on the attractivity of the radial front with speed $c$ are not clear without the assumption $f^{\prime}(M)<0$ (see Remark 1.4 below for the case $f^{\prime}(M)<0$ ). However, if $x_{0}$ denotes the point with respect to which the considered solution $u$ is radially symmetric and decreasing, and if $a$ is any fixed real number in $(0, M)$, then one knows from (1.14) that, for all $t$ large enough, there is a unique $\xi_{a}(t) \in \mathbb{R}$ such that $u\left(t, x_{0}+\xi_{a}(t) e\right)=a$ for all unit vectors $e$, and

$$
\limsup _{t \rightarrow+\infty}\left|\xi_{a}(t)-\xi(t)\right|<+\infty
$$

It is reasonable to conjecture that $u\left(t, x_{0}+\xi_{a}(t) e+x\right) \rightarrow \varphi\left(x \cdot e+\varphi^{-1}(a)\right)$ as $t \rightarrow+\infty$ locally uniformly in $x \in \mathbb{R}^{N}$ for any unit vector $e$, albeit the proof of this property would require different arguments from the ones used here.

Remark 1.4. In alternative (iii) of Theorem 1.1, if $M$ is further assumed to be nondegenerate, meaning here that $f^{\prime}(M)<0$, then there are $x_{0} \in \mathbb{R}^{N}$ and $\tau \in \mathbb{R}$ depending on $u$, such that

$$
\sup _{x \in \mathbb{R}^{N}}\left|u(t, x)-\varphi\left(\left|x-x_{0}\right|-c t+\frac{N-1}{c} \ln t+\tau\right)\right| \rightarrow 0 \text { as } t \rightarrow+\infty .
$$

\footnotetext{
${ }^{1}$ On the other hand, if $f$ were assumed to be monotone, namely nonincreasing, in a left neighborhood of $M$, then it would follow from [3, 64] that $\max _{|x| \leq c^{\prime} t}|u(t, x)-M| \rightarrow 0$ and $\max _{|x| \geq c^{\prime \prime} t} u(t, x) \rightarrow 0$ as $t \rightarrow+\infty$ for every $0 \leq c^{\prime}<c<c^{\prime \prime}$. This in particular yields (1.14), namely $\lim _{t \rightarrow+\infty} \xi(t) / t=c$, but this does not show property (1.13) on the boundedness of the radial width of the transition between 0 and $M$ at large times.
} 
In particular, property (1.13) then holds with $\xi(t)=c t-((N-1) / c) \ln t$ (say for $t \geq 1)$ if $f^{\prime}(M)<0$. Property (1.19) makes the position $\xi(t)$ and the limit profile of $u$ in all directions exactly known up to an $o(1)$ term as $t \rightarrow+\infty$. When 0 and $M$ are nondegenerate and $f$ has a single zero in the interval $(0, M)$, formula (1.19) for the solutions converging locally to $M$ follows from [68, Corollary 2] (see also [65] for more precise estimates on the position of the front at large times, and [35] for earlier but less precise estimates). It is easily seen from [68 that the proof extends to the case when 0 and $M$ are still nondegenerate and $f$ has more than one zero in $(0, M)$, since the unique profile $\varphi$ given in (1.15), with unique speed $c>0$, still satisfies $\varphi^{\prime}<0$ in $\mathbb{R}$ and converges exponentially to 0 and $M$ at $\pm \infty$.

Finally, let us comment the assumption (1.3) on $u$. It is essential in the derivation of (1.12) saying that the $\alpha$-limit set of the considered solutions is included in $\mathcal{E}$. If, instead of (1.3), one only assumes that $u(t, x) \rightarrow 0$ as $|x| \rightarrow+\infty$ for each $t \leq 0$ (and then, equivalently, for each $t \in \mathbb{R}$ ), but without any uniformity with respect to $t \leq 0$, then the conclusion does not hold in general. For instance, consider any $b>0$ and a function $f$ which is of the bistable type on $[0, b]$, namely there is $a \in(0, b)$ such that

$$
\left\{\begin{array}{l}
f(0)=f(a)=f(b)=0, \quad f^{\prime}(0)<0, \quad f^{\prime}(b)<0, \quad f^{\prime}(a)>0, \\
f<0 \text { in }(0, a), \quad f>0 \text { in }(a, b) .
\end{array}\right.
$$

The equation (1.1) with $f$ satisfying (1.20) was originally proposed in 2, 49] and is accordingly often called the Allen-Cahn equation or the Nagumo equation. It arises in a wide variety of contexts such as phase transition, combustion, ecology and many models of biology. If $f$ satisfies (1.20) and if

$$
\int_{0}^{b} f(s) d s>0
$$

then there are entire solutions $u: \mathbb{R} \times \mathbb{R} \rightarrow(0, b)$ of (1.1) in dimension $N=1$ that satisfy $\lim _{x \rightarrow \pm \infty} u(t, x)=0$ for each $t \in \mathbb{R}$ and behave as two further and further pulses as $t \rightarrow-\infty$, see [41. Namely, there is a solution $\phi: \mathbb{R} \rightarrow\left(0, a^{\prime}\right]$ of (1.4), where $a^{\prime} \in(a, b)$ is such that $F\left(a^{\prime}\right)=0$ and $\phi$ is the unique, up to shifts, solution of (1.4) ranging in $[0, b]$. The solutions $u$ constructed in [41] are such that

$$
u(t, x)-\phi(-\xi(t))-\phi(\xi(t)) \rightarrow 0 \text { as } t \rightarrow-\infty \text { uniformly in } x \in \mathbb{R},
$$

with $\lim _{t \rightarrow-\infty} \xi(t)=+\infty$. Therefore, these solutions $u$ do not satisfy (1.12). On the other hand, if $f$ satisfies (1.20) and

$$
\int_{0}^{b} f(s) d s<0
$$

then there are entire solutions $u: \mathbb{R} \times \mathbb{R} \rightarrow(0, b)$ of (1.1) that satisfy $\lim _{x \rightarrow \pm \infty} u(t, x)=0$ for each $t \in \mathbb{R}$ and behave as two far fronts as $t \rightarrow-\infty$, see [30]. Namely, under (1.20) and (1.22), equation (1.1) admits a traveling front $\varphi(x-c t)$ with $c<0$ and such that $\varphi: \mathbb{R} \rightarrow(0, b)$ is decreasing with $\varphi(-\infty)=b$ and $\varphi(+\infty)=0$, that is, $\varphi$ obeys (1.15) with $M=b$. The solutions $u$ constructed in [30] satisfy

$$
u(t, x)-\varphi(x-c t)-\varphi(-x-c t)+b \rightarrow 0 \text { as } t \rightarrow-\infty \text { uniformly in } x \in \mathbb{R},
$$


and also $\|u(t, \cdot)\|_{L^{\infty}(\mathbb{R})} \rightarrow 0$ as $t \rightarrow+\infty$. Since in this case there is $b^{\prime} \in(b,+\infty)$ such that $F<0$ in $\left(0, b^{\prime}\right]$, equation (1.4) does not admit any solution $\phi: \mathbb{R} \rightarrow\left[0, b^{\prime}\right]$, and the solutions $u$ then do not satisfy (1.12).

Remark 1.5. In Theorem 1.1, the assumption (1.3) can nevertheless be relaxed, still keeping the uniformity with respect to $t \leq 0$. More precisely, let any $\eta>0$ be such that $f<0$ in $(0, \eta]$. Notice that such a real number $\eta$ exists by (1.2). It then turns out that Theorem 1.1 holds if (1.3) is replaced by the assumption

$$
\limsup _{|x| \rightarrow+\infty}\left(\sup _{t \leq 0} u(t, x)\right) \leq \eta
$$

or $\lim \sup _{|x| \rightarrow+\infty}\left(\sup _{t \leq t_{0}} u(t, x)\right) \leq \eta$ for some $t_{0} \in \mathbb{R}$. As a matter of fact, (1.23) implies (and is then equivalent to) (1.3). Indeed, assume by way of contradiction that (1.23) holds, but not (1.3). Then there is a sequence $\left(t_{n}, x_{n}\right)_{n \in \mathbb{N}}$ in $(-\infty, 0] \times \mathbb{R}^{N}$ such that

$$
\lim _{n \rightarrow+\infty}\left|x_{n}\right|=+\infty \text { and } 0<\liminf _{n \rightarrow+\infty} u\left(t_{n}, x_{n}\right) \leq \limsup _{n \rightarrow+\infty} u\left(t_{n}, x_{n}\right) \leq \eta .
$$

Furthermore, it follows from standard parabolic estimates that, up to extraction of a subsequence, the functions $u_{n}:(t, x) \mapsto u_{n}(t, x)=u\left(t+t_{n}, x+x_{n}\right)$ converge in $C_{t, x}^{1,2}$ locally in $\mathbb{R} \times \mathbb{R}^{N}$ to a nonnegative bounded solution $u_{\infty}$ of (1.1) such that $u_{\infty}(0,0)>0$ and $0 \leq u_{\infty} \leq \eta$ in $(-\infty, 0] \times \mathbb{R}^{N}$. Therefore, the maximum principle yields $u_{\infty}(t, x) \leq \zeta\left(t+t_{0}\right)$ for all $t_{0}>0$ and $(t, x) \in\left[-t_{0},+\infty\right) \times \mathbb{R}^{N}$, where $\zeta$ obeys $\zeta(0)=\eta$ and $\zeta^{\prime}(t)=f(\zeta(t))$ for all $t \geq 0$. In particular, $0<u_{\infty}(0,0) \leq \zeta\left(t_{0}\right)$ for all $t_{0}>0$. But $\zeta(+\infty)=0$ since $f<0$ in $(0, \eta]$ and $f(0)=0$, leading to a contradiction. Therefore, (1.3) could equivalently be replaced by (1.23) in Theorem 1.1, but we preferred to state Theorem 1.1 with (1.3) since this assumption is simpler to write and does not involve the additional introduction of a quantity $\eta$.

1.4. Existence of monotone heteroclinic connections. Let us now discuss the existence of heteroclinic connections between a steady state $\phi$ of (1.4) and the constant states 0 or $M$. The results described in the following paragraphs are quite standard and inspired by similar ones in bounded domains [40]. See also [24, Theorem 1.2]. We just sketch here the main steps of the proof of the existence of heteroclininc connections for the sake of completeness. So, consider any solution $\phi$ of (1.4). Since $\phi$ decays exponentially to 0 at infinity (see also Section 4.1), so do its first- and second-order partial derivatives, from standard elliptic estimates. In particular, $\phi \in H^{1}\left(\mathbb{R}^{N}\right)$ and $\phi_{x_{i}} \in H^{1}\left(\mathbb{R}^{N}\right)$ for each $1 \leq i \leq N$. Since each first-order partial derivative $\phi_{x_{i}}$ changes sign and satisfies the equation $\Delta \phi_{x_{i}}+f^{\prime}(\phi) \phi_{x_{i}}=0$ in $\mathbb{R}^{N}$, it follows that $\phi$ is a strictly unstable solution of (1.4), in the sense that, for all $R>0$ large enough, the principal eigenvalue $\lambda_{R}$ of the operator $-\Delta-f^{\prime}(\phi)$ in the open Euclidean ball $B_{R}$ with center 0 and radius $R$, with Dirichlet boundary condition on $\partial B_{R}$, is such that $\lambda_{R}<0$. Let $\varphi_{R} \in C^{2}\left(\overline{B_{R}}\right)$ be a principal eigenfunction associated to this operator, namely

$$
-\Delta \varphi_{R}-f^{\prime}(\phi) \varphi_{R}=\lambda_{R} \varphi_{R} \text { in } B_{R}, \quad \varphi_{R}>0 \text { in } B_{R}, \text { and } \varphi_{R}=0 \text { on } \partial B_{R} .
$$

Fix any $R>0$ large enough such that $\lambda_{R}<0$. There exists then $\varepsilon^{*}>0$ such that, for all $\varepsilon \in\left(0, \varepsilon^{*}\right)$, the $C^{2}\left(\overline{B_{R}}\right)$ function $\phi_{R, \varepsilon}:=\phi-\varepsilon \varphi_{R}$ satisfies $0<\phi_{R, \varepsilon} \leq \phi$ in $\overline{B_{R}}, \phi_{R, \varepsilon}=\phi$ 
on $\partial B_{R}$ and

$$
\Delta \phi_{R, \varepsilon}+f\left(\phi_{R, \varepsilon}\right)<0 \text { in } B_{R}
$$

Denote $\widetilde{\phi}_{R, \varepsilon}(x)=\phi_{R, \varepsilon}(x)$ if $x \in \overline{B_{R}}$ and $\widetilde{\phi}_{R, \varepsilon}(x)=\phi(x)$ if $x \in \mathbb{R}^{N} \backslash \overline{B_{R}}$. Thus, for any $\varepsilon \in\left(0, \varepsilon^{*}\right)$, the bounded and uniformly continuous function $\widetilde{\phi}_{R, \varepsilon}$ is a generalized strict supersolution of (1.4), and the solution $u^{\varepsilon}$ of the Cauchy problem

$$
\left\{\begin{aligned}
u_{t}^{\varepsilon} & =\Delta u^{\varepsilon}+f\left(u^{\varepsilon}\right) & & \text { in }(0,+\infty) \times \mathbb{R}^{N}, \\
u^{\varepsilon}(0, \cdot) & =\widetilde{\phi}_{R, \varepsilon} & & \text { in } \mathbb{R}^{N},
\end{aligned}\right.
$$

is strictly decreasing in $t$. Furthermore, it is truly globally defined (in $[0,+\infty) \times \mathbb{R}^{N}$ ) and satisfies $0<u^{\varepsilon}<\widetilde{\phi}_{R, \varepsilon} \leq \phi$ in $(0,+\infty) \times \mathbb{R}^{N}$ from the parabolic maximum principle. The monotonicity in time and standard parabolic estimates yield the existence of a $C^{2}\left(\mathbb{R}^{N}\right)$ solution $\phi^{\prime}$ of $\Delta \phi^{\prime}+f\left(\phi^{\prime}\right)=0$ in $\mathbb{R}^{N}$ such that $0 \leq \phi^{\prime}<\phi$ in $\mathbb{R}^{N}$. Since by [9] any two solutions of (1.4) can not be ordered, it follows from the strong elliptic maximum principle that $\phi^{\prime} \equiv 0$ in $\mathbb{R}^{N}$. Without loss of generality, one can assume that $\varepsilon^{*} \varphi_{R}(0)<\phi(0) / 2$. Hence, for any $\varepsilon \in\left(0, \varepsilon^{*}\right)$, one has $u^{\varepsilon}(0,0)>\phi(0) / 2$, and there is a time $t^{\varepsilon}>0$ such that

$$
u^{\varepsilon}\left(t^{\varepsilon}, 0\right)=\frac{\phi(0)}{2} \text {. }
$$

Notice that $t^{\varepsilon}$ is unique since $u^{\varepsilon}$ is continuous and decreasing in $t$. Furthermore, from the continuous dependence of the solutions of the Cauchy problem associated to (1.1) with respect to the initial data, and since $\phi$ is a steady state, one infers that $t^{\varepsilon} \rightarrow+\infty$ as $\varepsilon \rightarrow 0$. Consider now a decreasing sequence $\left(\varepsilon_{n}\right)_{n \in \mathbb{N}}$ in $\left(0, \varepsilon^{*}\right)$ and converging to 0 , and define

$$
u_{n}(t, x)=u^{\varepsilon_{n}}\left(t+t^{\varepsilon_{n}}, x\right) \text { for }(t, x) \in\left[-t^{\varepsilon_{n}},+\infty\right) \times \mathbb{R}^{N} .
$$

From the previous observations and standard parabolic estimates, the functions $u_{n}$ converge up to extraction of a subsequence in $C_{t, x}^{1,2}$ locally in $\mathbb{R} \times \mathbb{R}^{N}$ to a solution $u_{\infty}$ of (1.1), such that $0 \leq u_{\infty} \leq \phi$ and $\left(u_{\infty}\right)_{t} \leq 0$ in $\mathbb{R} \times \mathbb{R}^{N}$, together with $u_{\infty}(0,0)=\phi(0) / 2$. Hence,

$$
0<u_{\infty}<\phi \text { in } \mathbb{R} \times \mathbb{R}^{N}
$$

from the strong maximum principle, and there are two steady states $\phi_{ \pm} \in C^{2}\left(\mathbb{R}^{N}\right)$ such that $u_{\infty}(t, \cdot) \rightarrow \phi_{ \pm}$as $t \rightarrow \pm \infty$ locally uniformly in $\mathbb{R}^{N}$ (and then uniformly since $0<u_{\infty}<\phi$ in $\mathbb{R} \times \mathbb{R}^{N}$ and $\phi(x) \rightarrow 0$ as $\left.|x| \rightarrow+\infty\right)$, with $0 \leq \phi_{+} \leq \phi_{-} \leq \phi$ and $\phi_{+}(0) \leq \phi(0) / 2 \leq \phi_{-}(0)$. The strong maximum principle and the non-existence of ordered solutions of (1.4) imply that $\phi_{-} \equiv \phi$ and $\phi_{+} \equiv 0$ in $\mathbb{R}^{N}$. Lastly, $\left(u_{\infty}\right)_{t}<0$ from the strong parabolic maximum principle applied to this function. In other words, the solution $u_{\infty}$ of (1.1) is a time-decreasing heteroclinic connection from $\phi$ to 0 .

Similarly, the function $\phi+\varepsilon \varphi_{R} \in C^{2}\left(\overline{B_{R}}\right)$ satisfies $\Delta\left(\phi+\varepsilon \varphi_{R}\right)+f\left(\phi+\varepsilon \varphi_{R}\right)>0$ in $B_{R}$ for all $\varepsilon>0$ small enough, with $R>0$ fixed large enough as above. Assume here that $M_{\phi}$, defined in (1.9), is a real number. Notice that the arguments of [9] based on the maximum principle and the sliding method imply that, for any classical positive solution $\phi^{\prime}$ of $\Delta \phi^{\prime}+f\left(\phi^{\prime}\right)=0$ in $\mathbb{R}^{N}$ such that $\phi^{\prime} \geq \phi$, one has either $\phi^{\prime} \equiv \phi$ in $\mathbb{R}^{N}$, or $\phi^{\prime} \geq \max _{\mathbb{R}^{N}} \phi$ and then $\phi^{\prime} \geq M_{\phi}$ in $\mathbb{R}^{N}$ (remember that $f>0$ in $\left[\max _{\mathbb{R}^{N}} \phi, M_{\phi}\right)$ ). Therefore, it follows that, for all $\varepsilon>0$ 
small enough, the solutions $u^{\varepsilon}$ of (1.25), with this time

$$
\widetilde{\phi}_{R, \varepsilon}= \begin{cases}\phi+\varepsilon \varphi_{R} & \text { in } \overline{B_{R}}, \\ \phi & \text { in } \mathbb{R}^{N} \backslash \overline{B_{R}},\end{cases}
$$

are increasing in time in $(0,+\infty) \times \mathbb{R}^{N}$, and satisfy $u^{\varepsilon}(t, \cdot) \rightarrow M_{\phi}$ as $t \rightarrow+\infty$ locally uniformly in $\mathbb{R}^{N}$. Furthermore, since $\phi$ is radially symmetric (with respect to, say, the origin without loss of generality) and decreasing in $|x|$, with $\Delta \phi(0)=-f(\phi(0))=-f\left(\max _{\mathbb{R}^{N}} \phi\right)<0$, and since the principal eigenfunction $\varphi_{R}$ of (1.24) is itself radially symmetric by uniqueness, it follows that the functions $\widetilde{\phi}_{R, \varepsilon}$ given in (1.26) are also radially symmetric and decreasing in $|x|$, for all $\varepsilon>0$ small enough. So are the functions $u^{\varepsilon}(t, \cdot)$ for all $t>0$. With the same arguments as in the previous paragraph, by defining a time $t^{\varepsilon}>0$ such that

$$
u^{\varepsilon}\left(t^{\varepsilon}, 0\right)=\frac{\phi(0)+M_{\phi}}{2},
$$

one infers the existence of a time-increasing heteroclinic connection between $\phi$ and $M_{\phi}$ (the convergence to $M_{\phi}$ being this time only locally uniform in $\mathbb{R}^{N}$ as $t \rightarrow+\infty$ ).

For bistable functions $f$ of the type (1.20)-(1.21), we also refer to [34, 46] for the existence of other time-increasing heteroclinic connections $u\left(t, x_{1}, x_{2}\right)=U\left(x_{1}, x_{2}-\gamma t\right)$ of (1.1), for any $\gamma>0$ large enough, between the extended one-dimensional solution $\phi\left(x_{1}, x_{2}\right)=\phi\left(x_{1}\right)$ of (1.4) and the constant $b=M_{\phi}$, in the sense that $u\left(t, x_{1}, x_{2}\right) \rightarrow \phi\left(x_{1}\right)$ as $t \rightarrow-\infty$ uniformly in $x_{1}$ and locally uniformly in $x_{2}$, and $u\left(t, x_{1}, x_{2}\right) \rightarrow b$ as $t \rightarrow+\infty$ locally uniformly in $\left(x_{1}, x_{2}\right)$. These connections are however not localized, that is, they do not satisfy (1.3): as a matter of fact, one has $\lim \sup _{\left|\left(x_{1}, x_{2}\right)\right| \rightarrow+\infty} u\left(t, x_{1}, x_{2}\right)=b>0$ for each $t \in \mathbb{R}$.

Assume now that $M_{\phi}=+\infty$. In that case, the solutions $u^{\varepsilon}$ of the previous paragraphs can not stay bounded and therefore blow up in finite or infinite time, according to the behavior of $f(s)$ as $s \rightarrow+\infty$. As above, without loss of generality, for all $\varepsilon>0$ small enough, the functions $u^{\varepsilon}(t, \cdot)$ are radially symmetric and decreasing in $|x|$ for all $t$ in their interval $\left(0, T^{\varepsilon}\right)$ of existence, hence $u^{\varepsilon}(t, 0) \rightarrow+\infty$ as $t \rightarrow T^{\varepsilon}$. For all $\varepsilon>0$ small enough, there is then a time $t^{\varepsilon} \in\left(0, T^{\varepsilon}\right)$ such that

$$
u^{\varepsilon}\left(t^{\varepsilon}, 0\right)=\phi(0)+1,
$$

and $t^{\varepsilon} \rightarrow+\infty$ as $\varepsilon \rightarrow 0$. Therefore, using again the strong parabolic maximum principle, there is a time-increasing solution $u_{\infty}$ of (1.1), defined now in $(-\infty, T) \times \mathbb{R}^{N}$ with $T \in$ $(0,+\infty]$, such that $u_{\infty}(0,0)=\phi(0)+1, u_{\infty}$ is radially symmetric and decreasing with respect to $|x|, u_{\infty}(t, \cdot) \rightarrow \phi$ uniformly in $\mathbb{R}^{N}$ as $t \rightarrow-\infty$, and $u_{\infty}(t, 0) \rightarrow+\infty$ as $t \rightarrow T$. In other words, $u_{\infty}$ blows up at time $T$ (which may be finite or infinite, according to the function $f$ ).

Lastly, we point out that, since any two solutions of (1.4) can not be ordered [9], it follows that (1.1) can not have any time-monotone heteroclinic connection between two different solutions $\phi_{ \pm}$of (1.4). However, the existence of non-time-monotone heteroclinic connections is not a priori ruled out.

Remark 1.6. For any positive bounded solution $u$ of (1.1) satisfying (1.3), the action

$$
E[u(t, \cdot)]=\int_{\mathbb{R}^{N}}\left(\frac{|\nabla u(t, x)|^{2}}{2}-F(u(t, x)) d x\right.
$$


is well defined and it is a Lyapunov functional, that is, $t \mapsto E[u(t, \cdot)]$ is non-increasing in $\mathbb{R}$ and even decreasing unless $u$ does not depend on $t$. We refer to Section 4.1 for more details. Notice that $E[\phi]>0=E[0]$ for any solution $\phi$ of (1.4) (this can be viewed as a consequence of the aforementioned existence of heteroclinic connections between $\phi$ and 0$)$. If $M_{\phi}$ is a real number and $u$ is a heteroclinic connection between $\phi$ and $M_{\phi}$, or more generally speaking in case (iii) of Theorem 1.1, then $E[u(t, \cdot)] \rightarrow-\infty$ as $t \rightarrow+\infty$. If $u$ is a heteroclinic connection between two different solutions $\phi_{ \pm}$of (1.4) in the sense that $\left\|u(t, \cdot)-\phi_{ \pm}\right\|_{L^{\infty}\left(\mathbb{R}^{N}\right)} \rightarrow 0$ as $t \rightarrow \pm \infty$ (this is a particular case of alternative (ii) of Theorem 1.1), then

$$
E\left[\phi_{-}\right]>E\left[\phi_{+}\right]
$$

Lastly, if a positive bounded solution $u$ of (1.1) satisfying (1.3) does not converge to a single solution of (1.4) as $t \rightarrow-\infty$, in the sense that there are at least two different solutions $\phi$ and $\phi^{\prime}$ of (1.4) such that

$$
\left\|u\left(t_{n}, \cdot\right)-\phi\right\|_{L^{\infty}\left(\mathbb{R}^{N}\right)} \rightarrow 0 \text { and }\left\|u\left(t_{n}^{\prime}, \cdot\right)-\phi^{\prime}\right\|_{L^{\infty}\left(\mathbb{R}^{N}\right)} \rightarrow 0 \text { as } n \rightarrow+\infty
$$

with $\lim _{n \rightarrow+\infty} t_{n}=\lim _{n \rightarrow+\infty} t_{n}^{\prime}=-\infty$, then $E[\phi]=E\left[\phi^{\prime}\right]$. Notice that in that situation, $\phi$ and $\phi^{\prime}$ are necessarily radially symmetric with respect to the same origin, since so is $u$, and by connectedness of the $\alpha$-limit set of $u$ there is then a continuum of such limit steady states as $t \rightarrow-\infty$ in the $\alpha$-limit set of $u$, all having the same Lagrangian (we also refer to the discussion before Corollary 2.3 below). As a consequence, if the Lagrangian $E$ is one-to-one of the set the solutions of (1.4) which are symmetric with respect to the same point, then there is a single $\phi \in \mathcal{E}$ such that $\|u(t, \cdot)-\phi\|_{L^{\infty}\left(\mathbb{R}^{N}\right)} \rightarrow 0$ as $t \rightarrow-\infty$.

\section{Some COROLlaries AND PARTICUlAR CASES}

In this section, we list some corollaries of Theorem 1.1 which correspond to further assumptions or to some special cases. In particular, the conclusion (1.12) will be made more precise under further assumptions.

2.1. Dimension $N=1$. The first corollary is concerned with the dimension $N=1$. In this case, the solutions of (1.4) are unique, up to shifts. Indeed, for any such solution $\phi$, it follows from (1.5) and (1.8) that $F<0$ in $\left(0, \max _{\mathbb{R}} \phi\right)=(0, \Phi(0))$ and $\Phi^{\prime}(r)=-\sqrt{-2 F(\Phi(r))}$ for all $r \geq 0$, hence the radial profile $\Phi$ is unique from the Cauchy-Lipshitz theorem. Furthermore, $F(\Phi(0))=0$ and $f(\Phi(0))>0$. One can also infer from (1.8) that, if there is $\beta \in(0,+\infty)$ such that $F<0$ in $(0, \beta)$ with $F(\beta)=0$ and $f(\beta)>0$ (notice that the hypotheses $F<0$ in $(0, \beta)$ and $F(\beta)=0$ imply that $f(\beta) \geq 0$ ), then (1.4) has a (unique up to shifts) solution.

Corollary 2.1. Assume that $N=1$, that $f$ satisfies (1.2) and that there is $\beta \in(0,+\infty)$ such that $F<0$ in $(0, \beta)$ with $F(\beta)=0$ and $f(\beta)>0$. Then $\mathcal{E} \neq \emptyset$ and, for any positive bounded solution $u$ of (1.1) satisfying (1.3), there is $\phi \in \mathcal{E}$ such that $\|u(t, \cdot)-\phi\|_{L^{\infty}(\mathbb{R})} \rightarrow 0$ as $t \rightarrow-\infty$. Furthermore, either $\|u(t, \cdot)\|_{L^{\infty}(\mathbb{R})} \rightarrow 0$ as $t \rightarrow+\infty$, or $u(t, x) \equiv \phi(x)$ in $\mathbb{R} \times \mathbb{R}$, or else the alternative (iii) of Theorem 1.1 holds.

Corollary 2.1 easily follows from Theorem 1.1, the previous observations and the fact that the solutions $u$ are necessarily even in $x$ with respect to some real number. The fact that 
case (ii) reduces to $u \equiv \phi$ is a consequence of the existence of a Lyapunov functional and the uniqueness of the solutions of (1.4) up to shifts. We refer to Section 5 for more details.

2.2. Non-existence of positive bounded solutions. In dimension $N=1$, with (1.2), the existence of a smallest positive root $\beta$ of $F$ with $f(\beta)>0$ is a necessary and sufficient condition for the existence of a solution of (1.4). In dimension $N \geq 2$, any solution $\phi$ of (1.4) satisfies $F\left(\max _{\mathbb{R}^{N}} \phi\right)>0$. Therefore, the next result immediately follows from Theorem 1.1 and the strong maximum principle.

Corollary 2.2. Assume that $f$ satisfies (1.2). In dimension $N=1$, if $F<0$ in $(0,+\infty)$ or if $F<0$ in $(0, \beta)$ with $F(\beta)=0$ and $f(\beta)=0$, then the only nonnegative bounded solution $u$ of (1.1) satisfying (1.3) is the trivial solution $u \equiv 0$ in $\mathbb{R} \times \mathbb{R}$. In dimension $N \geq 2$, if $F \leq 0$ in $[0,+\infty)$, then the same conclusion holds.

The assumptions of Corollary 2.2 are simple conditions ruling out the existence of solutions to (1.4). These assumptions are however not optimal in dimensions $N \geq 2$. For instance, if $N \geq 3$, for any positive real numbers $\gamma$ and $\delta$ and for any $p \geq(N+2) /(N-2)$, the equation (1.4) with

$$
f(s)=-\gamma s+\delta s^{p}
$$

does not admit any solution [36, 52. The same property holds with

$$
f(s)=-\gamma s-\delta s^{p}+\eta s^{q}
$$

with $N \geq 3, \gamma>0, \delta>0, \eta>0$, and $1<p \leq(N+2) /(N-2) \leq q$ or $(N+2) /(N-2)<p<q$, see [36, 50, 52]. In these two examples, Theorem 1.1 implies that the only nonnegative bounded solution $u$ of (1.1) satisfying (1.3) is then the trivial solution $u \equiv 0$ in $\mathbb{R} \times \mathbb{R}^{N}$.

On the other hand, much work has been devoted to the existence of solutions to (1.4) for some classes of functions $f$ satisfying (1.2), see the book [36]. For instance, if $N \geq 3$ and $\sup _{[0,+\infty)} F>0$ together with $\max (f(s), 0)=o\left(s^{(N+2) /(N-2)}\right)$ as $s \rightarrow+\infty$, then (1.4) admits solutions, see [6, [7, 67]. The existence holds in dimension $N=2$ if for instance $f$ satisfies (1.20)-(1.21), see [8], or if $\sup _{[0,+\infty)} F>0$ and $\max (f(s), 0)=o\left(e^{\alpha s^{2}}\right)$ as $s \rightarrow+\infty$ for all $\alpha>0$, see [4].

2.3. Discreteness or uniqueness up to shifts of the localized steady states. In Theorem 1.1, property (1.12) says that the solution $u$ is close to the family of steady states of (1.4) as $t \rightarrow-\infty$, that is, the $\alpha$-limit set of $u$ (with respect to the uniform convergence in $\mathbb{R}^{N}$ ) consists of solutions of (1.4), which turn out to be all symmetric with respect to a same point in $\mathbb{R}^{N}$, since so is $u$. Any two different solutions of (1.4) can not be ordered by [9], but it is not clear in general to know whether $u$ emanates from a single steady state or from a continuum of them (the possible existence of continua of solutions of (1.4) which are symmetric with respect to the same point is a difficult issue in general dimensions $N \geq 2$ ). However, under some further assumptions on the set of steady states, combined with the connectedness of the $\alpha$-limit sets of the solutions, one can be sure that a single state is selected. 
Corollary 2.3. Assume that $f$ satisfies (1.2) and (1.11), and that the set of solutions of (1.4) which are radially symmetric with respect to the origin is discrete. 2 Let $u$ be a positive bounded solution of (1.1) satisfying (1.3). Then there is $\phi \in \mathcal{E}$ such that $\|u(t, \cdot)-\phi\|_{L^{\infty}\left(\mathbb{R}^{N}\right)} \rightarrow 0$ as $t \rightarrow-\infty$. Furthermore,

(i) either $u(t, \cdot) \rightarrow 0$ uniformly in $\mathbb{R}^{N}$ as $t \rightarrow+\infty$,

(ii) or there is $\phi^{\prime} \in \mathcal{E}$ such that $u(t, \cdot) \rightarrow \phi^{\prime}$ uniformly in $\mathbb{R}^{N}$ as $t \rightarrow+\infty$, and, if $\phi=\phi^{\prime}$, then $u(t, x) \equiv \phi(x) \equiv \phi^{\prime}(x)$ in $\mathbb{R} \times \mathbb{R}^{N}$,

(iii) or else the alternative (iii) of Theorem 1.1 holds.

In the particular case when the solutions $\phi$ of (1.4) are unique up to shifts, then $F \leq 0$ in $\left[0, m_{\phi}\right]$ since otherwise, by applying the results of [7, 8 ] to the function $f$ extended by 0 in $\left(m_{\phi},+\infty\right)$, there would exist other solutions $\phi^{\prime}$ of (1.4) such that

$$
\max _{\mathbb{R}^{N}} \phi^{\prime}<m_{\phi}<\max _{\mathbb{R}^{N}} \phi .
$$

Hence, condition (1.11) is necessarily fulfilled if the solutions of (1.4) are unique up to shifts. Therefore, since the bounded positive solutions $u$ of (1.1) satisfying (1.3) are necessarily radially symmetric and decreasing with respect to a single point in $\mathbb{R}^{N}$, the following corollary immediately holds.

Corollary 2.4. Assume that $f$ satisfies (1.2) and that the solutions of (1.4) exist and are unique up to shifts. Let $u$ be a positive bounded solution of (1.1) satisfying (1.3). Then there is $\phi \in \mathcal{E}$ such that $\|u(t, \cdot)-\phi\|_{L^{\infty}\left(\mathbb{R}^{N}\right)} \rightarrow 0$ as $t \rightarrow-\infty$. Moreover, either $\|u(t, \cdot)\|_{L^{\infty}\left(\mathbb{R}^{N}\right)} \rightarrow 0$ as $t \rightarrow+\infty$, or $u(t, x) \equiv \phi(x)$ in $\mathbb{R} \times \mathbb{R}^{N}$, or else the alternative (iii) of Theorem 1.1 holds.

The existence and uniqueness up to shifts of the solutions of (1.4) is known for some classes of functions $f$. For instance, if $f$ satisfies (1.2) and if there are $0<a<a^{\prime}<b \leq+\infty$ such that $f<0$ in $(0, a), f>0$ in $(a, b), F\left(a^{\prime}\right)=0, f \leq 0$ in $[b,+\infty)$ and

$$
s \mapsto \frac{f(s)}{s-a^{\prime}} \text { is nonincreasing in }\left(a^{\prime}, b\right),
$$

then there exists a unique up to shifts solution $\phi$ of (1.4), see [23, 51] (in this case, $m_{\phi}=a$ and $\left.M_{\phi}=b\right)$. Notice that the monotonicity condition (2.3) is especially fulfilled if $f$ is nonincreasing in $\left[a^{\prime}, b\right)$. The condition (2.3) is not optimal for the uniqueness, since there are bistable functions $f$ satisfying the above conditions but (2.3) for which the uniqueness up to shifts holds for (1.4) in any dimension $N \geq 1$ (see especially the cubic functions $f$ of the type (2.9) below used in Corollary 2.7). The uniqueness up to shifts of the solutions of (1.4) also holds if $f$ satisfies (1.2) and if there is $a \in(0,+\infty)$ such that $f \leq 0$ in $[0, a], f>0$ in $(a,+\infty)$ and $s \mapsto s f^{\prime}(s) / f(s)$ is nonincreasing in $(a,+\infty)$, see [1, 66]. An example is the function $f$ given in (2.1), namely

$$
f(s)=-\gamma s+\delta s^{p}
$$

with $\gamma>0, \delta>0$ and $p>1$. As a matter of fact, for that function, the existence and uniqueness up to shifts of a solution $\phi$ of (1.4) holds if and only if $N \leq 2$, or $N \geq 3$

\footnotetext{
${ }^{2}$ This means that, for any radially symmetric solution $\phi$ of (1.4), there is $\varepsilon>0$ such that $\|\psi-\phi\|_{L^{\infty}\left(\mathbb{R}^{N}\right)} \geq \varepsilon$ for every radially symmetric solution $\psi$ of (1.4).
} 
and $1<p<(N+2) /(N-2)$, see also [6, 7, 8, 10, 11, 12, 36, 37, 44, 45, 52, 69]. In this case, one has $m_{\phi}=(\gamma / \delta)^{1 /(p-1)}$ and $M_{\phi}=+\infty$, and it follows from Corollary 2.4 that any positive bounded solution of (1.1) satisfying (1.3) is either independent of $t$ or converges to 0 uniformly in $\mathbb{R}^{N}$ as $t \rightarrow+\infty$. Another important example is that of functions $f$ of the type (2.2), namely

$$
f(s)=-\gamma s-\delta s^{p}+\eta s^{q},
$$

with $\gamma>0, \delta>0, \eta>0, p \neq q$, and $\min (p, q)>1$. The uniqueness up to shifts of the solutions $\phi$ of (1.4) holds in that case, and the existence holds if and only if $N \leq 2$, or $p<q<(N+2) /(N-2)$ with $N \geq 3$ (in this case, $M_{\phi}=+\infty$ and the bounded solutions of (1.1) satisfying (1.3) are either independent of $t$ or converge to 0 uniformly in $\mathbb{R}^{N}$ as $t \rightarrow+\infty$ ), or $p>q$ with $N \geq 3$ and $\beta$ is small enough (in this case, $M_{\phi}<+\infty$ ), see [66].

On the other hand, without (2.3) or the aforementioned conditions listed in the previous paragraph, some examples of non-uniqueness up to shifts in $\mathbb{R}^{N}$ with $N \geq 2$ are known, for functions $f$ of the bistable type (1.20) with $f<0$ in $(b,+\infty)$, see [51, or for some functions $f$ having one single positive zero, see [53] (notice that conditions (1.2) and (1.11) are automatically fulfilled for the functions considered in [51, 53]). In [13], for functions $f$ of the type $f(s)=-s+s^{p}+\lambda s^{q}$ with $\lambda>0$ large, $1<q<3$ and $p<5$ close to 5 (conditions (1.2) and (1.11) then hold), it was shown that (1.4) in dimension $N=3$ admits at least three solutions which are radially symmetric with respect to the origin. Furthermore, it is reasonable to conjecture from the proof given in [13] that the set of all such solutions is discrete, in which case Corollary 2.3 can be applied.

2.4. Bistable and cubic functions $f$. We complete this section by considering the class of bistable functions $f$ satisfying (1.20) for some $0<a<b$, namely

$$
\left\{\begin{array}{l}
f(0)=f(a)=f(b)=0, \quad f^{\prime}(0)<0, \quad f^{\prime}(b)<0, \quad f^{\prime}(a)>0, \\
f<0 \text { in }(0, a), \quad f>0 \text { in }(a, b),
\end{array}\right.
$$

together with

$$
f<0 \text { in }(b,+\infty)
$$

On the one hand, if

$$
\int_{0}^{b} f(s) d s \leq 0
$$

then $F<0$ in $(0, b) \cup(b,+\infty)$, hence Corollary 2.2 immediately yields the following result.

Corollary 2.5. If $f$ satisfies (2.4)-(2.6), then, in any dimension $N \geq 1$, the only nonnegative bounded solution $u$ of (1.1) satisfying (1.3) is the trivial solution $u \equiv 0$ in $\mathbb{R} \times \mathbb{R}^{N}$.

On the other hand, if

$$
\int_{0}^{b} f(s) d s>0
$$


then there are solutions $\phi$ of (1.4) [7, 8], and

$$
a=m_{\phi}<\max _{\mathbb{R}^{N}} \phi<M_{\phi}=b .
$$

It is also well known [3, 20] that there is a unique $c \in \mathbb{R}$, which is positive, and a unique up to shift function $\varphi \in C^{2}(\mathbb{R})$ such that

$$
\varphi^{\prime \prime}+c \varphi^{\prime}+f(\varphi)=0 \text { in } \mathbb{R}, \quad \varphi^{\prime}<0 \text { in } \mathbb{R}, \quad \varphi(-\infty)=b, \quad \varphi(+\infty)=0 .
$$

An immediate corollary of Corollary 2.4, Theorem 1.1 and property (1.19) in Remark 1.4 is the following result.

Corollary 2.6. Assume that $f$ satisfies (2.4) and (2.7) and that the solutions of (1.4) are unique up to shifts. Let $u$ be a positive bounded solution of (1.1) satisfying (1.3). Then there is $\phi \in \mathcal{E}$ such that $\|u(t, \cdot)-\phi\|_{L^{\infty}\left(\mathbb{R}^{N}\right)} \rightarrow 0$ as $t \rightarrow-\infty$. Moreover, either $\|u(t, \cdot)\|_{L^{\infty}\left(\mathbb{R}^{N}\right)} \rightarrow 0$ as $t \rightarrow+\infty$, or $u(t, x) \equiv \phi(x)$ in $\mathbb{R} \times \mathbb{R}^{N}$, or else there are $x_{0} \in \mathbb{R}^{N}$ and $\tau \in \mathbb{R}$ such that

$$
\sup _{x \in \mathbb{R}^{N}}\left|u(t, x)-\varphi\left(\left|x-x_{0}\right|-c t+\frac{N-1}{c} \ln t+\tau\right)\right| \rightarrow 0 \text { as } t \rightarrow+\infty
$$

where $c>0$ and $\varphi \in C^{2}\left(\mathbb{R}^{N}\right)$ are given in (2.8) .

Consider finally an important example of functions $f$ satisfying (1.20), namely the cubic nonlinearities

$$
f(s)=s(b-s)(s-a)
$$

with $0<a<b$. Notice that (2.7) is fulfilled if and only if $a<b / 2$. Furthermore, in that case, the solutions of (1.4) exist and are unique up to shifts, by [7, 8, 66]. As a consequence, the following corollary holds.

Corollary 2.7. Let $0<a<b$ and $f$ be of the type (2.9). If $a \geq b / 2$, then, in any dimension $N \geq 1$, the only nonnegative bounded solution $u$ of (1.1) satisfying (1.3) is the trivial solution $u \equiv 0$ in $\mathbb{R} \times \mathbb{R}^{N}$. If $a<b / 2$, then (1.4) admits solutions and, for any positive bounded solution $u$ of (1.1) satisfying (1.3), there is $\phi \in \mathcal{E}$ such that $\|u(t, \cdot)-\phi\|_{L^{\infty}\left(\mathbb{R}^{N}\right)} \rightarrow 0$ as $t \rightarrow-\infty$, and either $\|u(t, \cdot)\|_{L^{\infty}\left(\mathbb{R}^{N}\right)} \rightarrow 0$ as $t \rightarrow+\infty$, or $u(t, x) \equiv \phi(x)$ in $\mathbb{R} \times \mathbb{R}^{N}$, or else there are $x_{0} \in \mathbb{R}^{N}$ and $\tau \in \mathbb{R}$ such that

$$
\sup _{x \in \mathbb{R}^{N}}\left|u(t, x)-\varphi\left(\left|x-x_{0}\right|-c t+\frac{N-1}{c} \ln t+\tau\right)\right| \rightarrow 0 \text { as } t \rightarrow+\infty,
$$

where $c>0$ and $\varphi \in C^{2}\left(\mathbb{R}^{N}\right)$ are given in (2.8) .

Outline of the paper. Section 3 is concerned with some preliminary results on the existence of planar traveling fronts connecting 0 and $M$ (these fronts are used in alternative (iii) of Theorem 1.1) and on the existence of steady states in large balls under some assumptions on the nonlinearity. Section 4 is devoted to the proof of Theorem 1.1 and Section 5 to the proof of Corollaries 2.1 and 2.3 (the other corollaries follow from the other results, as already emphasized). 


\section{Some PRELIminary faCtS}

We start with the existence and uniqueness of traveling fronts $(c, \varphi)$ solving (1.15) and arising in alternative (iii) of Theorem 1.1 .

Lemma 3.1. Let $0<m<M$ and $f$ be a $C^{1}([0, M])$ function such that $f(0)=f(M)=0$, $f^{\prime}(0)<0, f>0$ in $(m, M), F<0$ in $(0, m]$ and $F(M)>0$. Then there are a unique $c \in \mathbb{R}$ and $\varphi: \mathbb{R} \rightarrow(0, M)$ of class $C^{2}(\mathbb{R})$ solving

$$
\varphi^{\prime \prime}+c \varphi^{\prime}+f(\varphi)=0 \text { in } \mathbb{R}, \varphi(-\infty)=M, \text { and } \varphi(+\infty)=0,
$$

where the uniqueness of $\varphi$ is understood up to shifts. Furthermore, $c>0$ and $\varphi^{\prime}<0$ in $\mathbb{R}$.

The result is expected since it has been well known under some additional assumptions on $f$. However we are not aware of a suitable reference for its proof, which is therefore sketched here for the sake of completeness.

Proof. First of all, the uniqueness of a pair $(c, \varphi)$ is a direct consequence of [20, Corollary 2.3] and the property $\varphi^{\prime}<0$ in $\mathbb{R}$ follows from [20, Lemma 2.1]. Furthermore, $c>0$ by integrating (3.1) against $\varphi^{\prime}$ over $\mathbb{R}$ and using the assumption $F(M)>0$.

Let us now show the existence of a pair $(c, \varphi)$ solving (3.1). From the assumptions made on $f$, it is easy to check that there are a decreasing sequence $\left(\varepsilon_{n}\right)_{n \in \mathbb{N}}$ in $(0, m)$ converging to 0 , and a sequence $\left(\bar{f}_{n}\right)_{n \in \mathbb{N}}$ such that each function

$$
\bar{f}_{n}:\left[\varepsilon_{n}, M+\varepsilon_{n}\right] \rightarrow \mathbb{R}
$$

is of class $C^{1}\left(\left[\varepsilon_{n}, M+\varepsilon_{n}\right]\right)$, the sequence $\left(\left\|\bar{f}_{n}\right\|_{C^{1}\left(\left[\varepsilon_{n}, M+\varepsilon_{n}\right]\right)}\right)_{n \in \mathbb{N}}$ is bounded, and for each $n \in \mathbb{N}$, there holds: $\bar{f}_{n}\left(\varepsilon_{n}\right)=\bar{f}_{n}\left(M+\varepsilon_{n}\right)=0, \bar{f}_{n}^{\prime}\left(\varepsilon_{n}\right)<0, \bar{f}_{n}^{\prime}\left(M+\varepsilon_{n}\right)<0$, the zeroes of $\bar{f}_{n}$ are all non-degenerate (that is, $\left.\left\{s \in\left[\varepsilon_{n}, M+\varepsilon_{n}\right]: \bar{f}_{n}(s)=\bar{f}_{n}^{\prime}(s)=0\right\}=\emptyset\right)$ ), together with $\bar{f}_{n} \geq \bar{f}_{n^{\prime}}$ in $\left[\varepsilon_{n}, M+\varepsilon_{n^{\prime}}\right]$ if $n \leq n^{\prime}, \bar{f}_{n} \geq f$ in $\left[\varepsilon_{n}, M\right]$, and $\max _{\left[\varepsilon_{n}, M\right]}\left|\bar{f}_{n}-f\right| \rightarrow 0$ as $n \rightarrow+\infty$. Furthermore, even if it means considering a subsequence, one can always assume that, for each $n \in \mathbb{N}$,

$$
\bar{f}_{n}>0 \text { in }\left(m, M+\varepsilon_{n}\right), \int_{\varepsilon_{n}}^{s} \bar{f}_{n}(\sigma) d \sigma<0 \text { for all } s \in\left(\varepsilon_{n}, m\right], \text { and } \int_{\varepsilon_{n}}^{M+\varepsilon_{n}} \bar{f}_{n}(\sigma) d \sigma>0 .
$$

For each $n \in \mathbb{N}$, it then follows from [20, Theorem 2.8] that there are $p \in \mathbb{N}$, some real numbers $\varepsilon_{n}=a_{0}<a_{1}<\cdots<a_{p}=M+\varepsilon_{n}$ and

$$
c_{1} \geq \cdots \geq c_{p}
$$

such that, for each $j \in\{1, \cdots, p\}$, there is a $C^{2}(\mathbb{R})$ function $\varphi_{j}: \mathbb{R} \rightarrow\left(a_{j-1}, a_{j}\right)$ satisfying

$$
\varphi_{j}^{\prime \prime}+c_{j} \varphi_{j}^{\prime}+\bar{f}_{n}\left(\varphi_{j}\right)=0 \text { in } \mathbb{R}, \varphi_{j}^{\prime}<0 \text { in } \mathbb{R}, \varphi_{j}(-\infty)=a_{j} \text {, and } \varphi_{j}(+\infty)=a_{j-1} .
$$

Notice that the quantities $p, a_{j}, c_{j}$ and $\varphi_{j}$ actually depend on $n$, that $\bar{f}_{n}\left(a_{j}\right)=0$ for all $0 \leq j \leq p$, and that the family $\left(c_{j}, \varphi_{j}\right)_{1 \leq j \leq p}$ is then a stacked combination of traveling fronts with non-increasing speeds for the reaction term $\bar{f}_{n}$. Since $a_{p-1} \leq m$ and $\int_{a_{p-1}}^{M+\varepsilon_{n}} \bar{f}_{n}(\sigma) d \sigma>0$ by (3.2), integrating (3.4) with $j=p$ against $\varphi_{p}^{\prime}$ over $\mathbb{R}$ implies that $c_{p}>0$. Furthermore, 
if $p \geq 2$, one would have $a_{1} \leq m$ and then $c_{1}<0$ by using again (3.2) and (3.4) with $j=1$, contradicting (3.3). Therefore, $p=1$ and there is then a solution $\left(\bar{c}_{n}, \bar{\varphi}_{n}\right)$ of

$$
\bar{\varphi}_{n}^{\prime \prime}+\bar{c}_{n} \bar{\varphi}_{n}^{\prime}+\bar{f}_{n}\left(\bar{\varphi}_{n}\right)=0 \text { in } \mathbb{R}, \bar{\varphi}_{n}^{\prime}<0 \text { in } \mathbb{R}, \bar{\varphi}_{n}(-\infty)=M+\varepsilon_{n}, \text { and } \bar{\varphi}_{n}(+\infty)=\varepsilon_{n},
$$

with $\bar{c}_{n}>0$.

Now, if $n<n^{\prime}$, then $M+\varepsilon_{n}>M+\varepsilon_{n^{\prime}}>\varepsilon_{n}>\varepsilon_{n^{\prime}}$ and, up to shifts, one has $\bar{\varphi}_{n} \geq \bar{\varphi}_{n^{\prime}}$ in $\mathbb{R}$ with equality at a point $\xi$ such that $\bar{\varphi}_{n}(\xi)=\bar{\varphi}_{n^{\prime}}(\xi) \in\left(\varepsilon_{n}, M+\varepsilon_{n^{\prime}}\right)$. On the other hand, if $\bar{c}_{n} \leq \bar{c}_{n^{\prime}}$, then one would have

$$
\bar{\varphi}_{n}^{\prime \prime}+\bar{c}_{n^{\prime}} \bar{\varphi}_{n}^{\prime}+\bar{f}_{n^{\prime}}\left(\bar{\varphi}_{n}\right) \leq \bar{\varphi}_{n}^{\prime \prime}+\bar{c}_{n} \bar{\varphi}_{n}^{\prime}+\bar{f}_{n}\left(\bar{\varphi}_{n}\right)=0=\bar{\varphi}_{n^{\prime}}^{\prime \prime}+\bar{c}_{n^{\prime}} \bar{\varphi}_{n^{\prime}}^{\prime}+\bar{f}_{n^{\prime}}\left(\bar{\varphi}_{n^{\prime}}\right)
$$

in the open interval $I=\left\{x \in \mathbb{R}: \varepsilon_{n}<\bar{\varphi}_{n}(x)<M+\varepsilon_{n^{\prime}}\right\}$. In other words, the functions $\bar{\varphi}_{n}$ and $\bar{\varphi}_{n^{\prime}}$ are respectively a super-solution and a solution of the same elliptic equation in $I$, with $\bar{\varphi}_{n} \geq \bar{\varphi}_{n^{\prime}}$ in $\mathbb{R} \supset I$. Since $\xi \in I$, it then follows from the strong maximum principle that $\bar{\varphi}_{n}(x)=\bar{\varphi}_{n^{\prime}}(x)$ for all $x \in I$. Since $I$ is of the type $I=(\zeta,+\infty)$ for some $\zeta \in \mathbb{R}$, one gets a contradiction by passing to the limit in $\bar{\varphi}_{n}(x)=\bar{\varphi}_{n^{\prime}}(x)$ as $x \rightarrow+\infty$. As a consequence, $\bar{c}_{n}>\bar{c}_{n^{\prime}}$ and the sequence $\left(\bar{c}_{n}\right)_{n \in \mathbb{N}}$ is decreasing, with $\bar{c}_{n}>0$ for every $n \in \mathbb{N}$.

Finally, there is $c \in \mathbb{R}$ such that $\bar{c}_{n} \rightarrow c \geq 0$ as $n \rightarrow+\infty$. Let $\eta$ be any real number in $(0, m)$ such that $f<0$ in $(0, \eta]$. Up to shifts, one can assume without loss of generality that $\bar{\varphi}_{n}(0)=\eta$ for all $n$ large enough (such that $\varepsilon_{n}<\eta$ ). From standard elliptic estimates, the functions $\bar{\varphi}_{n}$ converge in $C_{l o c}^{2}(\mathbb{R})$ to a $C^{2}(\mathbb{R})$ function $\varphi$ such that $\varphi(0)=\eta, 0 \leq \varphi \leq M$ in $\mathbb{R}, \varphi^{\prime} \leq 0$ in $\mathbb{R}$, and

$$
\varphi^{\prime \prime}+c \varphi^{\prime}+f(\varphi)=0 \text { in } \mathbb{R} .
$$

From standard elliptic estimates, $\varphi^{\prime}( \pm \infty)=\varphi^{\prime \prime}( \pm \infty)=f(\varphi( \pm \infty))=0$ and the choice of $\eta$ yields $\varphi(+\infty)=0$, hence $\varphi^{\prime}<0$ in $\mathbb{R}$ from the strong maximum principle and $0<\varphi(-\infty) \leq$ $M$. Integrating (3.5) against $\varphi^{\prime}$ over $\mathbb{R}$ implies that $F(\varphi(-\infty))=c \int_{\mathbb{R}}\left(\varphi^{\prime}\right)^{2} \geq 0$. Therefore, the assumptions on $f$ imply that $\varphi(-\infty)=M$. In other words, the pair $(c, \varphi)$ solves (3.1), and the proof of Lemma 3.1 is thereby complete.

Remark 3.2. The arguments used in the proof of Lemma 3.1 also lead to the approximation of the unique speed $c$ from below. Namely, as above, there are a decreasing sequence $\left(\varepsilon_{n}\right)_{n \in \mathbb{N}}$ in $(0, m)$ converging to 0 , and a sequence $\left(\underline{f}_{n}\right)_{n \in \mathbb{N}}$ such that each function

$$
\underline{f}_{n}:\left[-\varepsilon_{n}, M-\varepsilon_{n}\right] \rightarrow \mathbb{R}
$$

is of class $C^{1}\left(\left[-\varepsilon_{n}, M-\varepsilon_{n}\right]\right)$, the sequence $\left(\left\|\underline{f}_{n}\right\|_{C^{1}\left(\left[-\varepsilon_{n}, M-\varepsilon_{n}\right]\right)}\right)_{n \in \mathbb{N}}$ is bounded, and for each $n \in \mathbb{N}$, there holds: $\underline{f}_{n}\left(-\varepsilon_{n}\right)=\underline{f}_{n}\left(M-\varepsilon_{n}\right)=0, \underline{f}_{n}^{\prime}\left(-\varepsilon_{n}\right)<0, \underline{f}_{n}^{\prime}\left(M-\varepsilon_{n}\right)<0$, the zeroes of $\underline{f}_{n}$ are all non-degenerate, together with $\underline{f}_{n} \leq \underline{f}_{n^{\prime}}$ in $\left[-\varepsilon_{n^{\prime}}, \bar{M}^{n}-\varepsilon_{n}\right]$ if $n \leq n^{\prime}$, $\underline{f}_{n} \leq f$ in $\left[0, \bar{M}-\varepsilon_{n}\right]$, and $\max _{\left[0, M-\varepsilon_{n}\right]}\left|\underline{f}_{n}-f\right| \rightarrow 0$ as $n \rightarrow+\infty$. Lastly, after fixing a real number $m^{\prime} \in(m, M)$ such that $F<0$ in $\left(0, m^{\prime}\right]$, one can assume without loss of generality that, for each $n \in \mathbb{N}$,

$$
\underline{f}_{n}>0 \text { in }\left[m^{\prime}, M-\varepsilon_{n}\right), \int_{-\varepsilon_{n}}^{s} \underline{f}_{n}(\sigma) d \sigma<0 \text { for all } s \in\left(-\varepsilon_{n}, m^{\prime}\right] \text {, and } \int_{-\varepsilon_{n}}^{M-\varepsilon_{n}} \underline{f}_{n}(\sigma) d \sigma>0 \text {. }
$$

As in the proof of Lemma 3.1 , one can then show that, for each $n \in \mathbb{N}$, there is a solution $\left(\underline{c}_{n}, \underline{\varphi}_{n}\right)$ of $\underline{\varphi}_{n}^{\prime \prime}+\underline{c}_{n} \underline{\varphi}_{n}^{\prime}+\underline{f}_{n}\left(\underline{\varphi}_{n}\right)=0$ in $\mathbb{R}, \underline{\varphi}_{n}^{\prime}<0$ in $\mathbb{R}, \underline{\varphi}_{n}(-\infty)=M-\varepsilon_{n}, \underline{\varphi}_{n}(+\infty)=-\varepsilon_{n}$, 
and $\underline{c}_{n}>0$. Furthermore, the sequence $\left(\underline{c}_{n}\right)_{n \in \mathbb{N}}$ is increasing, and $\underline{c}_{n}<c$ for all $n \in \mathbb{N}$, with the same arguments as in the proof of Lemma 3.1. Finally, there is $\underline{c} \leq c$ such that $\underline{c}_{n} \rightarrow \underline{c}$ as $n \rightarrow+\infty$, and there is a $C^{2}(\mathbb{R})$ solution $\underline{\varphi}$ of (3.1) with speed $\underline{c}$ instead of $c$. The uniqueness of $(c, \varphi)$ then yields $\underline{c}=c$, hence $\underline{c}_{n} \rightarrow c$ as $n \rightarrow+\infty$.

The second preliminary result is concerned with the existence of solutions of some semilinear elliptic equations in large balls.

Lemma 3.3. Let $\alpha<\beta$ be two real numbers and $g:[\alpha, \beta] \rightarrow \mathbb{R}$ be a $C^{1}([\alpha, \beta])$ function such that $g(\alpha)=g(\beta)=0$ and

$$
G(\beta):=\int_{\alpha}^{\beta} g(\sigma) d \sigma>\int_{\alpha}^{s} g(\sigma) d \sigma=: G(s) \text { for all } s \in[\alpha, \beta) .
$$

Then, for each $\nu \in(\alpha, \beta)$, there are $R>0$ and $a C^{2}\left(\overline{B_{R}}\right)$ function $\psi$ such that

$$
\left\{\begin{array}{rll}
\Delta \psi+g(\psi) & =0 & \text { in } \overline{B_{R}} \\
\alpha \leq \psi & <\beta \quad \text { in } \overline{B_{R}} \\
\psi & =\alpha & \text { on } \partial B_{R} \\
\max _{\overline{B_{R}}} \psi=\psi(0) & >\nu &
\end{array}\right.
$$

Proof. The proof is standard, based on [6], so we just sketch it. Let $\bar{g}: \mathbb{R} \rightarrow \mathbb{R}$ be the function defined by $\bar{g}(s)=g(s)$ for $s \in[\alpha, \beta]$, and $\bar{g}(s)=0$ for $s \in \mathbb{R} \backslash[\alpha, \beta]$, and let us still call $G$ the primitive of $\bar{g}$ vanishing at $\alpha$. For each $r>0$, there is a minimizer $\psi_{r} \in \alpha+H_{0}^{1}\left(B_{r}\right)$ of the Lagrangian $J_{r}$ defined in $\alpha+H_{0}^{1}\left(B_{r}\right)$ by

$$
J_{r}(\varphi)=\frac{1}{2} \int_{B_{r}}|\nabla \varphi(x)|^{2} d x-\int_{B_{r}} G(\varphi(x)) d x, \quad J_{r}\left(\psi_{r}\right)=\min _{\varphi \in \alpha+H_{0}^{1}\left(B_{r}\right)} J_{r}(\varphi),
$$

Owing to the definitions of $\bar{g}$ and $G$, one can assume without loss of generality that $\psi_{r}$ ranges in $[\alpha, \beta]$, hence $\psi_{r}$ is of class $C^{2}\left(\overline{B_{r}}\right)$ from elliptic estimates and it solves $\Delta \psi_{r}+g\left(\psi_{r}\right)=0$ in $\overline{B_{r}}$ with $\psi_{r}=\alpha$ on $\partial B_{r}$. The strong elliptic maximum principle also yields $\psi_{r}<\beta$ in $\overline{B_{r}}$ and, either $\psi_{r} \equiv \alpha$ in $\overline{B_{r}}$, or $\psi_{r}>\alpha$ in $B_{r}$. In both cases, $\psi_{r}$ is a radially symmetric and nonincreasing function of $|x|$ (from [26] in the latter). In particular, $\max _{\overline{B_{r}}} \psi_{r}=\psi_{r}(0) \in[\alpha, \beta)$.

Let us now show that

$$
\max _{\overline{B_{r}}} \psi_{r}=\psi_{r}(0) \rightarrow \beta \text { as } r \rightarrow+\infty,
$$

which will then provide $R>0$ and a solution $\psi$ of (3.7), given a fixed real number $\nu \in(\alpha, \beta)$. Assume by way of contradiction that there are $\theta \in(\alpha, \beta)$, a sequence $\left(r_{k}\right)_{k \in \mathbb{N}} \rightarrow+\infty$ and a sequence $\left(\psi_{r_{k}}\right)_{k \in \mathbb{N}}$ of $C^{2}\left(\overline{B_{r_{k}}}\right)$ functions such that each $\psi_{r_{k}}: \overline{B_{r_{k}}} \rightarrow[\alpha, \beta)$ minimizes $J_{r_{k}}$ in $\alpha+H_{0}^{1}\left(B_{r_{k}}\right)$ and $\max _{\overline{B_{r_{k}}}} \psi_{r_{k}}=\psi_{r_{k}}(0) \leq \theta<\beta$. From the assumptions made on $g$, there is $\delta>0$ such that $G(s) \leq G(\beta)-\delta$ for all $s \in[\alpha, \theta]$. Hence, $J_{r_{k}}\left(\psi_{r_{k}}\right) \geq(\delta-G(\beta)) \alpha_{N} r_{k}^{N}$ for all $k \in \mathbb{N}$, where $\alpha_{N}>0$ denotes the Lebesgue measure of the $N$-dimensional unit ball $B_{1}$. On the other hand, after assuming without loss of generality that $r_{k}>1$ for every $k \in \mathbb{N}$, consider the function $\varphi_{k} \in \alpha+H_{0}^{1}\left(B_{r_{k}}\right)$ defined by $\varphi_{k}(x)=\beta$ for $x \in B_{r_{k}-1}$ 
and $\varphi_{k}(x)=\alpha+(\beta-\alpha)\left(r_{k}-|x|\right)$ for $x \in \overline{B_{r_{k}}} \backslash B_{r_{k}-1}$. For each $k \in \mathbb{N}$, one has

$$
\begin{aligned}
J_{r_{k}}\left(\psi_{r_{k}}\right) & \leq J_{r_{k}}\left(\varphi_{k}\right)=\frac{\alpha_{N}}{2}\left(r_{k}^{N}-\left(r_{k}-1\right)^{N}\right)-G(\beta) \alpha_{N}\left(r_{k}-1\right)^{N}-\int_{B_{r_{k}} \backslash B_{r_{k}-1}} G\left(\varphi_{k}(x)\right) d x \\
& \leq \alpha_{N}\left(\frac{1}{2}+\max _{[\alpha, \beta]}|G|\right)\left(r_{k}^{N}-\left(r_{k}-1\right)^{N}\right)-G(\beta) \alpha_{N}\left(r_{k}-1\right)^{N} .
\end{aligned}
$$

This implies that

$$
\delta r_{k}^{N} \leq\left(\frac{1}{2}+\max _{[\alpha, \beta]}|G|+G(\beta)\right)\left(r_{k}^{N}-\left(r_{k}-1\right)^{N}\right) .
$$

It contradicts $\lim _{k \rightarrow+\infty} r_{k}=+\infty$, since $\delta>0$. As a consequence, (3.8) holds and the proof of Lemma 3.3 is thereby complete.

\section{Proof of Theorem 1.1}

This section is devoted to the proof of Theorem 1.1. Throughout it, one assumes that $f$ satisfies (1.2) and (1.11), and $u$ denotes a positive bounded solution of (1.1) satisfying (1.3). Sections 4.1 and 4.2 are concerned with the behaviors of $u$ as $t \rightarrow-\infty$ and $t \rightarrow+\infty$, respectively.

4.1. The behavior of $u$ as $t \rightarrow-\infty$. We here establish (1.12) and further integral properties of the solution $u$. First of all, it follows from [54, Theorem 1.1] that there is a point $x_{0} \in \mathbb{R}^{N}$ such that

$$
\begin{cases}u(t, x)=u(t, y) & \text { for all }(t, x, y) \in \mathbb{R} \times \mathbb{R}^{N} \times \mathbb{R}^{N} \text { with }\left|x-x_{0}\right|=\left|y-x_{0}\right|, \\ \nabla u(t, x) \cdot\left(x-x_{0}\right)<0 & \text { for all }(t, x) \in \mathbb{R} \times \mathbb{R}^{N} \text { with } x \neq x_{0} .\end{cases}
$$

Furthermore, [54, Corollary 2.5] implies that there are some positive constants $C$ and $\nu$ such that

$$
0<u(t, x) \leq C e^{-\nu|x|} \text { for all }(t, x) \in(-\infty, 0] \times \mathbb{R}^{N}
$$

Denote

$$
M_{0}=\|u\|_{L^{\infty}\left(\mathbb{R} \times \mathbb{R}^{N}\right)}>0
$$

and $L=\nu^{2}+\max _{\left[0, M_{0}\right]}\left|f^{\prime}\right|$. For any unit vector $e$ of $\mathbb{R}^{N}$, the function $\bar{u}(t, x)=C e^{-\nu x \cdot e+L t}$ satisfies $\bar{u}_{t}(t, x)-\Delta \bar{u}(t, x)-f(\bar{u}(t, x)) \geq 0$ for any $(t, x) \in \mathbb{R} \times \mathbb{R}^{N}$ such that $\bar{u}(t, x) \leq M_{0}$. Therefore, the maximum principle implies that $u(t, x) \leq \bar{u}(t, x)$ for all $(t, x) \in[0,+\infty) \times \mathbb{R}^{N}$ and for any unit vector $e$, hence $u(t, x) \leq C e^{-\nu|x|+L t}$ for all $(t, x) \in[0,+\infty) \times \mathbb{R}^{N}$. By combining this inequality with (4.2), for every $T \in \mathbb{R}$, there is a real number $C_{T}>0$ such that

$$
0<u(t, x) \leq C_{T} e^{-\nu|x|} \text { for all }(t, x) \in(-\infty, T] \times \mathbb{R}^{N} .
$$

From standard parabolic estimates, one also infers that, for every $T \in \mathbb{R}$, there is a real number $C_{T}^{\prime}>0$ such that

$$
u(t, x)+\left|u_{t}(t, x)\right|+|\nabla u(t, x)|+\sum_{1 \leq i, j \leq N}\left|u_{x_{i} x_{j}}(t, x)\right| \leq C_{T}^{\prime} e^{-\nu|x|} \text { for all }(t, x) \in(-\infty, T] \times \mathbb{R}^{N}
$$


In particular, the action

$$
E[u(t, \cdot)]=\int_{\mathbb{R}^{N}}\left(\frac{|\nabla u(t, x)|^{2}}{2}-F(u(t, x)) d x\right.
$$

is well defined at each time $t \in \mathbb{R}$, and Lebesgue's dominated convergence theorem implies that the function $t \mapsto E[u(t, \cdot)]$ is of class $C^{1}(\mathbb{R})$ with

$$
\frac{d}{d t} E[u(t, \cdot)]=-\int_{\mathbb{R}^{N}}\left(u_{t}(t, x)\right)^{2} d x \leq 0
$$

for every $t \in \mathbb{R}$. Furthermore, (4.4) yields $\sup _{t \leq T}|E[u(t, \cdot)]|<+\infty$ for every $T \in \mathbb{R}$, and there is then $\ell \in \mathbb{R}$ such that

$$
E[u(t, \cdot)] \rightarrow \ell \text { as } t \rightarrow-\infty .
$$

Consider now any sequence $\left(t_{n}\right)_{n \in \mathbb{N}}$ converging to $-\infty$, and denote

$$
u_{n}(t, x)=u\left(t+t_{n}, x\right)
$$

for $(t, x) \in \mathbb{R} \times \mathbb{R}^{N}$. From (4.4) and further standard parabolic estimates, there is a classical nonnegative bounded solution $u_{\infty}$ of (1.1) such that, up to extraction of a subsequence, $u_{n} \rightarrow u_{\infty}$ in $C_{t, x}^{1,2}$ locally in $\mathbb{R} \times \mathbb{R}^{N}$, together with

$$
\left\|u_{n}(t, \cdot)-u_{\infty}(t, \cdot)\right\|_{L^{\infty}\left(\mathbb{R}^{N}\right)} \rightarrow 0 \text { and } E\left[u_{n}(t, \cdot)\right] \rightarrow E\left[u_{\infty}(t, \cdot)\right] \text { as } n \rightarrow+\infty,
$$

for every $t \in \mathbb{R}$. Notice also that $u_{\infty}$ satisfies (4.4) with the constant $C_{0}^{\prime}$ in the whole set $\mathbb{R} \times \mathbb{R}^{N}$. Since $E\left[u_{n}(t, \cdot)\right]=E\left[u\left(t+t_{n}, \cdot\right)\right] \rightarrow \ell$ as $n \rightarrow+\infty$, for every $t \in \mathbb{R}$, one infers that $E\left[u_{\infty}(t, \cdot)\right]=\ell$ for every $t \in \mathbb{R}$, hence $\left(u_{\infty}\right)_{t} \equiv 0$ in $\mathbb{R} \times \mathbb{R}^{N}$ from (4.6) applied to $u_{\infty}$. As a consequence, $u_{\infty}$ is a bounded nonnegative steady state solving

$$
\left\{\begin{array}{l}
\Delta u_{\infty}+f\left(u_{\infty}\right)=0 \text { and } u_{\infty} \geq 0 \text { in } \mathbb{R}^{N}, \\
u_{\infty}(x) \rightarrow 0 \text { as }|x| \rightarrow+\infty .
\end{array}\right.
$$

From the elliptic maximum principle, it follows that either $u_{\infty} \equiv 0$ in $\mathbb{R}^{N}$, or $u_{\infty}>0$ in $\mathbb{R}^{N}$. In the former case, one has $u\left(t_{n}, \cdot\right)=u_{n}(0, \cdot) \rightarrow 0$ as $n \rightarrow+\infty$ uniformly in $\mathbb{R}^{N}$, hence $0<u\left(t_{n}, \cdot\right) \leq \eta$ in $\mathbb{R}^{N}$ for all $n$ large enough, where $\eta$ is a positive real number such that

$$
f<0 \text { in }(0, \eta] .
$$

Thus, for all $t \in \mathbb{R}$, one gets that $0<u(t, \cdot)=u\left(t-t_{n}+t_{n}, \cdot\right) \leq \zeta\left(t-t_{n}\right)$ in $\mathbb{R}^{N}$ for all $n$ large enough, where $\zeta$ obeys $\zeta(0)=\eta$ and $\zeta^{\prime}(t)=f(\zeta(t))$ for all $t \geq 0$. Since $\zeta(+\infty)=0$ and $\lim _{n \rightarrow+\infty} t_{n}=-\infty$, it follows that $u(t, \cdot) \leq 0$ in $\mathbb{R}^{N}$ for all $t \in \mathbb{R}$, a contradiction. Therefore, $u_{\infty}>0$ is a steady state solving (1.4), namely $u_{\infty} \in \mathcal{E}$. In particular, $\mathcal{E}$ is not empty.

Since in the previous paragraph the sequence $\left(t_{n}\right)_{n \in \mathbb{N}}$ converging to $-\infty$ was arbitrary, one concludes that

$$
\inf _{\phi \in \mathcal{E}}\|u(t, \cdot)-\phi\|_{L^{\infty}\left(\mathbb{R}^{N}\right)} \rightarrow 0 \text { as } t \rightarrow-\infty
$$

namely (1.12) has been proven. The observations of the previous paragraph also imply that

$$
E[\phi]=\ell
$$


for every $\phi \in \mathcal{E}$ belonging to the $\alpha$-limit set of $u$.

Remark 4.1. Remember that, from assumption (1.11), the map $(\emptyset \neq) \mathcal{E} \ni \phi \mapsto m_{\phi}$ takes a constant value, that is, there is $m>0$ such that

$$
m=m_{\phi}>0
$$

for all $\phi \in \mathcal{E}$. The quantity $M \in(m,+\infty]$ defined in (1.9) and (1.17)-(1.18) is such that

$$
f>0 \text { in }(m, M)
$$

from (1.10). In the present remark, we claim that

$$
0<u(t, x)<M \text { for all }(t, x) \in \mathbb{R} \times \mathbb{R}^{N} .
$$

By assumption, $u$ is positive. So there is nothing to show if $M=+\infty$. Assume now that $M<+\infty$. From the above proof of (1.12), there is $\phi \in \mathcal{E}$ and a sequence $\left(t_{n}\right)_{n \in \mathbb{N}}$ converging to $-\infty$ such that $\left\|u\left(t_{n}, \cdot\right)-\phi\right\|_{L^{\infty}\left(\mathbb{R}^{N}\right)} \rightarrow 0$ as $n \rightarrow+\infty$. Since $\max _{\mathbb{R}^{N}} \phi<M$ by (1.9) and (1.17), one has $u\left(t_{n}, \cdot\right)<M$ in $\mathbb{R}^{N}$ for all $n$ large enough. Since $f(M)=0$, it then follows from the maximum principle (applied for $n$ large enough) that, for each $t \in \mathbb{R}$, $u(t, \cdot)=u\left(t-t_{n}+t_{n}, \cdot\right)<M$ in $\mathbb{R}^{N}$, that is, (4.11) holds.

4.2. The behavior of $u$ as $t \rightarrow+\infty$. In the section, we consider the behavior of the entire solution $u$ as $t \rightarrow+\infty$. The proof is divided into five main steps.

Step 1: two key-lemmas. The proof of the dichotomy as $t \rightarrow+\infty$ between the uniformly localized solutions and the spreading solutions is based on two key-lemmas. The first one gives a sufficient condition for the finiteness and attractiveness of the quantity $M \in(m,+\infty]$ defined in (1.9) and (1.17)-(1.18).

Lemma 4.2. For every $\varepsilon>0$, there is a real number $\rho_{\varepsilon}>0$ such that, if

$$
u\left(t_{0}, \cdot\right) \geq m+\varepsilon \text { in } \overline{B\left(y_{0}, \rho_{\varepsilon}\right)}
$$

for some $\left(t_{0}, y_{0}\right) \in \mathbb{R} \times \mathbb{R}^{N}$, then

$$
M<+\infty
$$

and

$$
\max _{|x| \leq \gamma t}|u(t, x)-M| \rightarrow 0 \text { as } t \rightarrow+\infty
$$

for some $\gamma>03$

Remark 4.3. For a function $f$ such that $M$ is a priori assumed to be finite, the conclusion (4.13) can also be viewed as a consequence of [16. Lemma 2.4], which is based on the existence of approximated planar fronts defined in bounded intervals (see also [20, Theorem 3.2] and [3, Theorem 6.2] for related results with more specific nonlinearities $f$ in the one- and multi-dimensional cases). We here both show (4.13) and the finiteness of $M$ under assumption (4.12). Moreover, the proof of (4.13) given below differs from that of [16, Lemma 2.4] as it is based on Lemma 3.3 and on the existence of compactly supported steady states.

\footnotetext{
${ }^{3}$ We point out that, when $\varepsilon>M_{0}-m, \rho_{\varepsilon}$ can be arbitrary because (4.12) is not fulfilled in that case.
} 
Proof of Lemma 4.2. Let $\varepsilon>0$ be fixed throughout the proof. Assume first, by way of contradiction, that $M=+\infty$, and that there exists a sequence $\left(t_{n}, y_{n}\right)_{n \in \mathbb{N}}$ in $\mathbb{R} \times \mathbb{R}^{N}$ such that $u\left(t_{n}, \cdot\right) \geq m+\varepsilon$ in $\overline{B\left(y_{n}, n\right)}$. From (1.10), it then follows that $f>0$ in $(m,+\infty)$. From standard parabolic estimates, the functions

$$
u_{n}:(t, x) \mapsto u_{n}(t, x)=u\left(t+t_{n}, x+y_{n}\right)
$$

converge in $C_{t, x}^{1,2}$ locally in $\mathbb{R} \times \mathbb{R}^{N}$, up to extraction of a subsequence, to a nonnegative bounded solution $u_{\infty}$ of (1.1) such that $u_{\infty}(0, \cdot) \geq m+\varepsilon$ in $\mathbb{R}^{N}$. Hence, $u_{\infty}(t, \cdot) \geq \varsigma(t)$ in $\mathbb{R}^{N}$ for all $t \geq 0$, where $\varsigma$ obeys

$$
\left\{\begin{array}{l}
\varsigma^{\prime}(t)=f(\varsigma(t)) \text { for } t \geq 0, \\
\varsigma(0)=m+\varepsilon .
\end{array}\right.
$$

This is impossible since $u_{\infty}$ is bounded while $f>0$ in $[m+\varepsilon,+\infty)$. As a consequence, there is $\varrho_{\varepsilon}>0$ such that if

$$
u\left(t_{0}, \cdot\right) \geq m+\varepsilon \text { in } \overline{B\left(y_{0}, \varrho_{\varepsilon}\right)}
$$

for some $\left(t_{0}, y_{0}\right) \in \mathbb{R} \times \mathbb{R}^{N}$, then

$$
M<+\infty .
$$

We now claim that there is $\rho_{\varepsilon} \in\left[\varrho_{\varepsilon},+\infty\right)$ such that, if condition (4.12) is fulfilled for some $\left(t_{0}, y_{0}\right) \in \mathbb{R} \times \mathbb{R}^{N}$, then $M<+\infty$ (from the previous paragraph) and

$$
u(t, \cdot) \rightarrow M \text { as } t \rightarrow+\infty \text { locally uniformly in } \mathbb{R}^{N} \text {. }
$$

Assume not. Then there is a sequence $\left(\tau_{n}, z_{n}\right)_{n \in \mathbb{N}, n \geq \varrho_{\varepsilon}}$ in $\mathbb{R} \times \mathbb{R}^{N}$ such that $u\left(\tau_{n}, \cdot\right) \geq m+\varepsilon$ in $\overline{B\left(z_{n}, n\right)}$ (hence, $M<+\infty$ ) and

$$
u(t, \cdot) \not \rightarrow M \text { as } t \rightarrow+\infty \text { locally uniformly in } \mathbb{R}^{N} .
$$

Notice that (4.11) then implies that

$$
m<m+\varepsilon<M .
$$

On the other hand, since $F<0$ in $(0, m]$ by (1.11) and (4.10), since $F(M)>0$ by (1.17)(1.18) and since $f>0$ in $(m, M)$ by (1.10), one infers that $F(s)<F(M)$ for all $s \in[0, M)$. Lemma 3.3 applied with $\alpha=0, \beta=M, \gamma=m$ and $g=f$ yields the existence of $R>0$ and a $C^{2}\left(\overline{B_{R}}\right)$ function $\psi$ such that

$$
\left\{\begin{array}{rlrl}
\Delta \psi+f(\psi) & =0 & & \text { in } \overline{B_{R}} \\
0 \leq \psi & <M & & \text { in } \overline{B_{R}} \\
\psi & =0 & & \text { on } \partial B_{R} \\
M>\max _{\overline{B_{R}}} \psi=\psi(0) & >m>0 . &
\end{array}\right.
$$

Let $\varsigma$ be the solution of (4.14). Since $m+\varepsilon<M$ and $f>0$ in $(m, M)$ with $f(M)=0$, one has $\varsigma(t) \rightarrow M$ as $t \rightarrow+\infty$. Hence, there is a positive real number $T>0$ such that

$$
\varsigma(T)>\psi(0) .
$$

Up to extraction of a subsequence, the functions

$$
v_{n}:(t, x) \mapsto v_{n}(t, x)=u\left(t+\tau_{n}, x+z_{n}\right)
$$


converge in $C_{t, x}^{1,2}$ locally in $\mathbb{R} \times \mathbb{R}^{N}$ to a nonnegative bounded solution $v_{\infty}$ of (1.1) such that $v_{\infty}(0, \cdot) \geq m+\varepsilon$ in $\mathbb{R}^{N}$. Hence, $v_{\infty}(T, \cdot) \geq \varsigma(T)>\psi(0)$. It then follows from the last line in (4.17) that there is $n_{0} \in \mathbb{N}$ (with $n_{0} \geq \varrho_{\varepsilon}$ ) such that

$$
u\left(T+\tau_{n_{0}},+z_{n_{0}}\right)>\psi \text { in } \overline{B_{R}} .
$$

Let then $w$ be the solution of the equation $w_{t}=\Delta w+f(w)$ in $(0,+\infty) \times \mathbb{R}^{N}$ with initial condition given by

$$
w(0, x)= \begin{cases}\psi(x) & \text { if } x \in \overline{B_{R}}, \\ 0 & \text { if } x \in \mathbb{R}^{N} \backslash \overline{B_{R}} .\end{cases}
$$

Since $\psi$ satisfies (4.17) and $f(M)=f(0)=0$, the parabolic maximum principle implies that $0<w<M$ in $(0,+\infty) \times \mathbb{R}^{N}$ and $w$ is increasing with respect to $t$ in $[0,+\infty) \times \mathbb{R}^{N}$. From standard parabolic estimates and uniqueness of the limit, there is a $C^{2}\left(\mathbb{R}^{N}\right)$ solution $w_{\infty}$ of $\Delta w_{\infty}+f\left(w_{\infty}\right)=0$ in $\mathbb{R}^{N}$ with $0<w_{\infty} \leq M$ in $\mathbb{R}^{N}$ and $w_{\infty}>\psi$ in $\overline{B_{R}}$. Let then $e$ be any unit vector of $\mathbb{R}^{N}$. By continuity, there is $s_{0}>0$ such that $w_{\infty}>\psi(\cdot-s e)$ in $\overline{B(s e, R)}$ for all $s \in\left[0, s_{0}\right]$. Calling

$$
s^{*}=\sup \left\{s>0: w_{\infty}>\psi\left(\cdot-s^{\prime} e\right) \text { in } \overline{B\left(s^{\prime} e, R\right)} \text { for all } s^{\prime} \in[0, s]\right\} \in\left[s_{0},+\infty\right]
$$

and assuming that $s^{*}<+\infty$, one would have $w_{\infty} \geq \psi\left(\cdot-s^{*} e\right)$ in $\overline{B\left(s^{*} e, R\right)}$ with equality somewhere at a point $x^{*} \in \overline{B\left(s^{*} e, R\right)}$. Since $w_{\infty}>0$ in $\mathbb{R}^{N}$ and $\psi=0$ on $\partial B_{R}$, the point $x^{*}$ would be an interior point in $B\left(s^{*} e, R\right)$, hence $w_{\infty} \equiv \psi\left(\cdot-s^{*} e\right)$ in $\overline{B\left(s^{*} e, R\right)}$ from the strong maximum principle. This is impossible on $\partial B\left(s^{*}, R\right)$. Thus $s^{*}=+\infty$ and, since the unit vector $e$ was arbitrary, one gets that $(M \geq) w_{\infty}>\psi(0)>m$ in $\mathbb{R}^{N}$. The positivity of $f$ in $(m, M)$ then implies that $w_{\infty} \equiv M$ in $\mathbb{R}^{N}$, hence

$$
w(t, \cdot) \rightarrow M \text { as } t \rightarrow+\infty \text { locally uniformly in } \mathbb{R}^{N} .
$$

Together with (4.19)-(4.20) and the maximum principle, one infers that

$$
\liminf _{t \rightarrow+\infty}\left(\min _{K} u(t, \cdot)\right) \geq M
$$

for any compact set $K \subset \mathbb{R}^{N}$, and finally $u(t, \cdot) \rightarrow M$ as $t \rightarrow+\infty$ locally uniformly in $\mathbb{R}^{N}$ from (4.11). This contradicts (4.16).

As a conclusion of the previous paragraph, there is a positive real number $\rho_{\varepsilon}\left(\geq \varrho_{\varepsilon}\right)$ such that if condition (4.12) is fulfilled for some $\left(t_{0}, y_{0}\right) \in \mathbb{R} \times \mathbb{R}^{N}$, then $M<+\infty$ and (4.15) holds. Let us finally show that this implies the stronger property (4.13): $\max _{|x| \leq \gamma t}|u(t, x)-M| \rightarrow 0$ as $t \rightarrow+\infty$, for some $\gamma>0$. To do so, observe on the one hand that, since $w(t, \cdot) \rightarrow M$ as $t \rightarrow+\infty$ locally uniformly in $\mathbb{R}^{N}$ by (4.21) and since $\max _{\overline{B_{R}}} \psi<M$, there is a time $\tau>0$ such that

$$
w(\tau, \cdot) \geq \psi(\cdot-s e) \text { in } \overline{B(s e, R)} \text { for every unit vector } e \text { and every } s \in[0,1] .
$$

In other words, $w(\tau, \cdot) \geq w(0, \cdot-s e)$ in $\mathbb{R}^{N}$ for every unit vector $e$ and every $s \in[0,1]$. From the maximum principle, one gets that $w(2 \tau, \cdot) \geq w(\tau, \cdot-s e) \geq w(0, \cdot-2 s e)$ in $\mathbb{R}^{N}$ for every unit vector $e$ and every $s \in[0,1]$. Hence, by an immediate induction,

$$
w(k \tau, \cdot) \geq w(0, \cdot-s e) \text { in } \mathbb{R}^{N} \text { for every } k \in \mathbb{N} \text {, every unit vector } e \text {, and every } s \in[0, k] .
$$


On the other hand, (4.15) and (4.17) yield the existence of a time $\tau_{*}>0$ such that

$$
u\left(\tau_{*}, \cdot\right) \geq \psi \text { in } \overline{B_{R}},
$$

that is, $u\left(\tau_{*}, \cdot\right) \geq w(0, \cdot)$ in $\mathbb{R}^{N}$. Therefore,

$$
u\left(\tau_{*}+k \tau, \cdot\right) \geq w(0, \cdot-s e) \text { in } \mathbb{R}^{N}
$$

for every $k \in \mathbb{N}$, every unit vector $e$, and every $s \in[0, k]$. In particular,

$$
\min _{\overline{B_{k}}} u\left(\tau_{*}+k \tau, \cdot\right) \geq w(0,0)=\psi(0) \text { for all } k \in \mathbb{N} .
$$

We finally claim that

$$
\max _{|x| \leq t /(2 \tau)}|u(t, x)-M| \rightarrow 0 \text { as } t \rightarrow+\infty
$$

which will give the desired conclusion (4.13) with $\gamma=1 /(2 \tau)$. Assume by way of contradiction that (4.23) does not hold. Since $0<u<M$ in $\mathbb{R} \times \mathbb{R}^{N}$ by (4.11), there are then a real number $\theta \in[0, M)$ and a sequence $\left(s_{n}, \xi_{n}\right)_{n \in \mathbb{N}}$ in $(0,+\infty) \times \mathbb{R}^{N}$ such that

$$
\lim _{n \rightarrow+\infty} s_{n}=+\infty, \lim _{n \rightarrow+\infty} u\left(s_{n}, \xi_{n}\right)=\theta, \text { and }\left|\xi_{n}\right| \leq \frac{s_{n}}{2 \tau} \text { for all } n \in \mathbb{N} .
$$

Consider any integer $j \in \mathbb{N}$. For all $n$ large enough, write

$$
s_{n}=\tau_{*}+k_{n} \tau+s_{n}^{\prime}, \quad \text { with } k_{n} \in \mathbb{N} \text { and } s_{n}^{\prime} \in[j \tau,(j+1) \tau)
$$

(the quantities $k_{n}$ and $s_{n}^{\prime}$ depend on $j$ as well, but this does not matter). Up to extraction of a subsequence, there is $s_{\infty}^{\prime} \in[j \tau,(j+1) \tau]$ such that $s_{n}^{\prime} \rightarrow s_{\infty}^{\prime}$ as $n \rightarrow+\infty$. Up to extraction of another subsequence, the functions

$$
U_{n}:(t, x) \mapsto U_{n}(t, x)=u\left(t+\tau_{*}+k_{n} \tau, x+\xi_{n}\right)
$$

converge in $C_{t, x}^{1,2}$ locally in $\mathbb{R} \times \mathbb{R}^{N}$ to a solution $U_{\infty}$ of (1.1) such that $0 \leq U_{\infty} \leq M$ in $\mathbb{R} \times \mathbb{R}^{N}$. For each $x \in \mathbb{R}^{N}$, one has $\left|x+\xi_{n}\right| \leq k_{n}$ for all $n$ large enough, since $\left|\xi_{n}\right| \leq s_{n} /(2 \tau)$ for all $n$ and $s_{n} \sim k_{n} \tau$ as $n \rightarrow+\infty$ from (4.25) and $\lim _{n \rightarrow+\infty} s_{n}=+\infty$. It then follows from (4.22) that $U_{\infty}(0, \cdot) \geq \psi(0)$ in $\mathbb{R}^{N}$, hence

$$
U_{\infty}(t, \cdot) \geq \omega(t) \text { in } \mathbb{R}^{N} \text { for all } t \geq 0
$$

where $\omega$ obeys $\omega^{\prime}(t)=f(\omega(t))$ and $\omega(0)=\psi(0)$. Since $m<\psi(0)<M$ and $f>0$ in $(m, M)$ with $f(M)=0$, one has $\omega(t) \rightarrow M$ as $t \rightarrow+\infty$ (notice that $U_{\infty}$ and $s_{\infty}^{\prime} \in[j \tau,(j+1) \tau]$ depend on $j \in \mathbb{N}$, but $\psi(0)$ and $\omega$ do not). It also follows from (4.24)-(4.25) that $U_{\infty}\left(s_{\infty}^{\prime}, 0\right)=\theta<M$, hence $M>\theta \geq \omega\left(s_{\infty}^{\prime}\right)$. Since $s_{\infty}^{\prime} \in[j \tau,(j+1) \tau]$ and $\omega(+\infty)=M$, the passage to the limit as $j \rightarrow+\infty$ in the inequality $M>\theta \geq \omega\left(s_{\infty}^{\prime}\right)$ leads to a contradiction. As a conclusion, (4.23) has been shown and the proof of Lemma 4.2 is thereby complete.

The second key-lemma gives a quantitative estimate of the time the solution takes to go from $m+\varepsilon$ to any value $\lambda$ less than $M$ in large balls.

Lemma 4.4. Under the notations of Lemma 4.2, for every $\varepsilon>0, \lambda<M$ and $r \geq 0$, there are some real numbers $\rho_{\varepsilon, \lambda, r} \geq \rho_{\varepsilon}>0$ and $T_{\varepsilon, \lambda, r}>0$ such that, if

$$
u\left(t_{0}, \cdot\right) \geq m+\varepsilon \text { in } \overline{B\left(y_{0}, R\right)}
$$


for some $\left(t_{0}, y_{0}\right) \in \mathbb{R} \times \mathbb{R}^{N}$ and $R \geq \rho_{\varepsilon, \lambda, r}$, then

$$
u(t, \cdot) \geq \lambda \text { in } \overline{B\left(y_{0}, R+r\right)} \text { for all } t \geq t_{0}+T_{\varepsilon, \lambda, r} .
$$

Proof. Let us fix $\varepsilon>0, \lambda<M$ and $r \geq 0$, and let $\rho_{\varepsilon}>0$ be given by Lemma 4.2. Assume by way of contradiction that the conclusion of Lemma 4.4 does not hold. Then there exist two sequences $\left(R_{n}\right)_{n \in \mathbb{N}}$ and $\left(T_{n}\right)_{n \in \mathbb{N}}$ of positive real numbers converging to $+\infty$, and a sequence $\left(t_{n}, y_{n}, z_{n}\right)_{n \in \mathbb{N}}$ in $\mathbb{R} \times \mathbb{R}^{N} \times \mathbb{R}^{N}$ such that

$$
u\left(t_{n}, \cdot\right) \geq m+\varepsilon \text { in } \overline{B\left(y_{n}, R_{n}\right)}, z_{n} \in \overline{B\left(y_{n}, R_{n}+r\right)}, \text { and } u\left(t_{n}+T_{n}, z_{n}\right)<\lambda,
$$

for all $n \in \mathbb{N}$. Notice that Lemma 4.2 then implies that $M<+\infty$, and that $m+\varepsilon<M$ by (4.11) and (4.26).

Let now $R>0$ and $\psi \in C^{2}\left(\overline{B_{R}}\right)$ be as in (4.17), let $w$ be the solution of the Cauchy problem $w_{t}=\Delta w+f(w)$ in $(0,+\infty) \times \mathbb{R}^{N}$ with initial condition $w(0, \cdot)$ given by (4.20), and let $\varsigma \in C^{1}([0,+\infty))$ and $T>0$ be defined as in (4.14) and (4.18). For any $\varrho>0$, call $v_{\varrho}$ the solution of $\left(v_{\varrho}\right)_{t}=\Delta v_{\varrho}+f\left(v_{\varrho}\right)$ in $(0,+\infty) \times \mathbb{R}^{N}$ with initial condition $v_{\varrho}(0, \cdot)$ defined by:

$$
v_{\varrho}(0, x)= \begin{cases}m+\varepsilon & \text { if } x \in B_{\varrho}, \\ 0 & \text { if } x \in \mathbb{R}^{N} \backslash B_{\varrho} .\end{cases}
$$

From standard parabolic estimates, there holds $v_{\varrho}(T, \cdot) \rightarrow \varsigma(T)(>\psi(0))$ as $\varrho \rightarrow+\infty$ locally uniformly in $\mathbb{R}^{N}$ (e.g. see [31, Theorem 4.1]). Hence, there is $\varrho_{0}>0$ such that $v_{\varrho_{0}}(T, \cdot)>\psi(0)$ in $\overline{B_{R}}$, and then

$$
v_{\varrho_{0}}(T, \cdot)>w(0, \cdot) \text { in } \mathbb{R}^{N} \text {. }
$$

Since $\lambda<M$ by assumption and since $w(t, \cdot) \rightarrow M$ as $t \rightarrow+\infty$ locally uniformly in $\mathbb{R}^{N}$ by (4.21), there is $T^{\prime}>0$ such that

$$
w(t, \cdot) \geq \lambda \text { in } \overline{B_{r+\varrho_{0}}} \text { for all } t \geq T^{\prime} .
$$

Notice that the parameters and functions introduced in the previous paragraph do not depend on $n$. Coming back to (4.26), one can assume without loss of generality that $R_{n} \geq \varrho_{0}$ for all $n \in \mathbb{N}$. Hence, by (4.26), for each $n \in \mathbb{N}$, there is a point $y_{n}^{\prime}$ such that

$$
\left|z_{n}-y_{n}^{\prime}\right| \leq r+\varrho_{0} \text { and } B\left(y_{n}^{\prime}, \varrho_{0}\right) \subset B\left(y_{n}, R_{n}\right)
$$

and thus $u\left(t_{n}, \cdot\right) \geq v_{\varrho_{0}}\left(0, \cdot-y_{n}^{\prime}\right)$ in $\mathbb{R}^{N}$. The maximum principle then yields

$$
u\left(t_{n}+T, \cdot\right) \geq v_{\varrho_{0}}\left(T, \cdot-y_{n}^{\prime}\right)>w\left(0, \cdot-y_{n}^{\prime}\right) \text { in } \mathbb{R}^{N}
$$

and $u\left(t_{n}+t, \cdot\right)>w\left(t-T, \cdot-y_{n}^{\prime}\right)$ in $\mathbb{R}^{N}$ for all $t \geq T$. For all $n$ large enough so that $T_{n} \geq T+T^{\prime}$, it then follows that $u\left(t_{n}+T_{n}, z_{n}\right)>w\left(T_{n}-T, z_{n}-y_{n}^{\prime}\right) \geq \lambda$ by (4.27)-(4.28), contradicting the last property of (4.26).

To sum up, the existence of the sequences $\left(R_{n}\right)_{n \in \mathbb{N}},\left(T_{n}\right)_{n \in \mathbb{N}}$ and $\left(t_{n}, y_{n}, z_{n}\right)_{n \in \mathbb{N}}$ is ruled out and the proof of Lemma 4.4 is thereby complete.

Step 2: a dichotomy. Fix a real number $\eta>0$ such that (4.8) holds. Since $f(m)=f\left(m_{\phi}\right)=0$ for all $\phi \in \mathcal{E}$, with $m_{\phi}>0$, one has

$$
0<\eta<m \text {. }
$$

Define $g(s)=-f(-s)$ for all $s \in[-m, 0]$. From assumption (1.11), the $C^{1}([-m, 0])$ function $g$ satisfies (3.6) with $\alpha=-m$ and $\beta=0$. Lemma 3.3 applied with $\nu=-\eta \in(-m, 0)$ 
then provides the existence of $R_{1}>0$ and of a function $\psi \in C^{2}\left(\overline{B_{R_{1}}}\right)$ solving $\Delta \psi+g(\psi)=0$ and $-m \leq \psi<0$ in $\overline{B_{R_{1}}}$ with $\psi=-m$ on $\partial B_{R_{1}}$ and $\max _{\overline{B_{R_{1}}}} \psi=\psi(0)>-\eta$. In other words, the function $\varphi=-\psi \in C^{2}\left(\overline{B_{R_{1}}}\right)$ solves

$$
\left\{\begin{array}{rlrl}
\Delta \varphi+f(\varphi) & =0 & \text { in } \overline{B_{R_{1}}} \\
0<\varphi & \leq m \text { in } \overline{B_{R_{1}}} \\
\varphi & =m \text { on } \partial B_{R_{1}} \\
\frac{\min }{\bar{B}_{R_{1}}}=\varphi(0) & <\eta .
\end{array}\right.
$$

Furthermore, it follows from [26] that $\varphi$ is radially symmetric, namely there is a $C^{2}\left(\left[0, R_{1}\right]\right)$ function $\widetilde{\varphi}$ such that

$$
\varphi(x)=\widetilde{\varphi}(|x|) \text { for all } x \in \overline{B_{R_{1}}}
$$

and the Hopf lemma (or, here, the Cauchy-Lipschitz theorem) implies that

$$
\delta:=\widetilde{\varphi}^{\prime}\left(R_{1}\right)>0 .
$$

Since $u$ is bounded by assumption, it follows from standard parabolic estimates that there is a positive constant $M_{2}$ such that

$$
\left|u_{x_{i}, x_{j}}(t, x)\right| \leq M_{2} \text { for all }(t, x) \in \mathbb{R} \times \mathbb{R}^{N} \text { and } 1 \leq i, j \leq N .
$$

From Lemma 4.2 applied with $\varepsilon=\delta^{2} /\left(4 M_{2}\right)>0$, there is a real number

$$
R_{2}=\rho_{\varepsilon}=\rho_{\delta^{2} /\left(4 M_{2}\right)}>0
$$

such that, if $u\left(t_{0}, \cdot\right) \geq m+\delta^{2} /\left(4 M_{2}\right)$ in $\overline{B\left(y_{0}, R_{2}\right)}$ for some $\left(t_{0}, y_{0}\right) \in \mathbb{R} \times \mathbb{R}^{N}$, then $M<+\infty$ and $\max _{|x| \leq \gamma t}|u(t, x)-M| \rightarrow 0$ as $t \rightarrow+\infty$ for some $\gamma>0$. Here, by choosing $M_{2}$ large if necessary, we may assume that $\varepsilon=\delta^{2} /\left(4 M_{2}\right)<M-m$.

Remember now that $x_{0} \in \mathbb{R}^{N}$ is a center of symmetry given by (4.1) and that $u$ is localized for $t \leq 0$, in the sense of (1.3). There is then a point $x_{1} \in \mathbb{R}^{N}$ such that

$$
\left|x_{1}-x_{0}\right| \geq R_{2}+\frac{\delta}{2 M_{2}}+R_{1} \text { and } u(t, \cdot)<\varphi(0) \text { in } \overline{B\left(x_{1}, R_{1}\right)} \text { for all } t \leq 0 .
$$

We shall then compare $u$ with $\varphi\left(\cdot-x_{1}\right)$ in $\overline{B\left(x_{1}, R_{1}\right)}$. First of all, owing to (4.29), one has $u(t, \cdot)<\varphi\left(\cdot-x_{1}\right)$ in $\overline{B\left(x_{1}, R_{1}\right)}$ for all $t \leq 0$. Two cases may then occur:

$$
\text { either } u(t, \cdot)<\varphi\left(\cdot-x_{1}\right) \text { in } \overline{B\left(x_{1}, R_{1}\right)} \text { for all } t \in \mathbb{R} \text {, }
$$

or there is $t_{0} \in \mathbb{R}$ such that $u(t, \cdot)<\varphi\left(\cdot-x_{1}\right)$ in $\overline{B\left(x_{1}, R_{1}\right)}$ for all $t<t_{0}$

$$
\text { and } u\left(t_{0}, \cdot\right) \leq \varphi\left(\cdot-x_{1}\right) \text { in } \overline{B\left(x_{1}, R_{1}\right)} \text { with equality somewhere in } \overline{B\left(x_{1}, R_{1}\right)} \text {. }
$$

It will turn out that (4.34) will lead to the conclusions (i) or (ii) of Theorem 1.1, whereas (4.35) will lead to the spreading case (iii). We consider in Step 3 the alternative (4.34), while (4.35) will be dealt with in Steps 4 and 5 .

Step 3: convergence at large times if $u$ is uniformly localized. We assume here that (4.34) holds. Thus, $u\left(t, x_{1}\right)<\varphi(0)<\eta$ for all $t \in \mathbb{R}$ and property (4.1) implies that $u(t, x)<$ 
$\varphi(0)<\eta$ for all $(t, x) \in \mathbb{R} \times \mathbb{R}^{N}$ with $\left|x-x_{0}\right| \geq\left|x_{1}-x_{0}\right|$. The arguments used in Remark 1.5 then yield

$$
u(t, x) \rightarrow 0 \text { as }|x| \rightarrow+\infty \text { uniformly in } t \in \mathbb{R},
$$

that is, $u$ is uniformly localized. From [9, Theorem 1.1] (see also [22]) and standard parabolic estimates, it follows that either $u(t, \cdot) \rightarrow 0$ as $t \rightarrow+\infty$ in $H^{1}\left(\mathbb{R}^{N}\right) \cap C^{2}\left(\mathbb{R}^{N}\right)$ (that is, the alternative (i) holds in Theorem [1.1), or there is positive steady state $\phi \in \mathcal{E}$ solving (1.4) such that $u(t, \cdot) \rightarrow \phi$ as $t \rightarrow+\infty$ in $H^{1}\left(\mathbb{R}^{N}\right) \cap C^{2}\left(\mathbb{R}^{N}\right)$ (that is, the alternative (ii) holds in Theorem 1.1). Notice that, in the former case, the action $E[u(t, \cdot)]$ defined by (4.5) satisfies $E[u(t, \cdot)] \rightarrow E[0]=0$ as $t \rightarrow+\infty$, while in the latter case,

$$
E[u(t, \cdot)] \rightarrow E[\phi] \text { as } t \rightarrow+\infty .
$$

In all cases, the function $t \mapsto E[u(t, \cdot)]$ is then bounded in $\mathbb{R}$.

Step 4: the transition is radially bounded if u spreads, proof of (1.13). We assume in the sequel that (4.35) holds. We shall see that this case leads to the alternative (iii) of the conclusion of Theorem 1.1. We prove the property (1.13) in the present Step 4, and property (1.14) in Step 5. The proof of (1.13) is based on the maximum principle and on suitable estimates on the oscillations of the radial positions of the level sets of $u$ at large time, as well as on the key-lemmas of Step 1.

First of all, since $\varphi$ solves (4.29), the alternative (4.35) and the parabolic strong maximum principle imply that there is a point $x_{2} \in \partial B\left(x_{1}, R_{1}\right)$ such that

$$
u\left(t_{0}, x_{2}\right)=\varphi\left(x_{2}-x_{1}\right)=m .
$$

Because of (4.1), (4.35) and of the inequality $\left|x_{1}-x_{0}\right| \geq R_{2}+\delta /\left(2 M_{2}\right)+R_{1}>R_{1}$, together with the fact that $\varphi<m$ in $B_{R_{1}}$ (from the elliptic strong maximum principle), it turns out that $x_{2}$ is the unique point lying at the intersection of the sphere $\partial B\left(x_{1}, R_{1}\right)$ and the segment $\left[x_{0}, x_{1}\right]$. In particular,

$$
\left|x_{2}-x_{0}\right|=\left|x_{1}-x_{0}\right|-R_{1} \geq R_{2}+\frac{\delta}{2 M_{2}} .
$$

Furthermore, from (4.35) and the definitions of $\widetilde{\varphi}$ and $\delta$ satisfying (4.30)-(4.31), it follows that

$$
-\left|\nabla u\left(t_{0}, x_{2}\right)\right|=\nabla u\left(t_{0}, x_{2}\right) \cdot \frac{x_{2}-x_{0}}{\left|x_{2}-x_{0}\right|} \leq-\delta<0
$$

Together with (4.32) and (4.1) again, one infers that

$$
\nabla u\left(t_{0}, x\right) \cdot \frac{x-x_{0}}{\left|x-x_{0}\right|} \leq-\frac{\delta}{2}<0 \text { for all } x \text { such that }\left|x_{2}-x_{0}\right|-\frac{\delta}{2 M_{2}} \leq\left|x-x_{0}\right| \leq\left|x_{2}-x_{0}\right| \text {. }
$$

Since $u\left(t_{0}, \cdot\right)=m$ at the point $x_{2}$ and then on $\partial B\left(x_{0},\left|x_{2}-x_{0}\right|\right)$, it follows that

$$
u\left(t_{0}, \cdot\right) \geq m+\frac{\delta^{2}}{4 M_{2}} \text { on } \partial B\left(x_{0},\left|x_{2}-x_{0}\right|-\frac{\delta}{2 M_{2}}\right), \text { with }\left|x_{2}-x_{0}\right|-\frac{\delta}{2 M_{2}} \geq R_{2},
$$

hence

$$
u\left(t_{0}, \cdot\right) \geq m+\frac{\delta^{2}}{4 M_{2}} \text { in } \overline{B\left(x_{0}, R_{2}\right)}
$$


by (4.1) again. Lemma 4.2 and the definition (4.33) of $R_{2}$ then imply that

$$
M<+\infty
$$

and

$$
\max _{|x| \leq \gamma t}|u(t, x)-M| \rightarrow 0 \text { as } t \rightarrow+\infty
$$

for some $\gamma>0$.

Secondly, (4.1) together with (4.3) and (4.38) yield the existence of a real number $\tau_{1}$ such that

$$
\text { for each } t \geq \tau_{1},\left\{\begin{array}{l}
\max _{\mathbb{R}^{N}} u(t, \cdot)=u\left(t, x_{0}\right)>m \\
\text { there is a unique } \xi(t)>0 \text { such that } u(t, \cdot)=m \text { on } \partial B\left(x_{0}, \xi(t)\right),
\end{array}\right.
$$
and $\lim \inf _{t \rightarrow+\infty} \xi(t) / t \geq \gamma>0$. In particular,

$$
\lim _{t \rightarrow+\infty} \xi(t)=+\infty
$$

The implicit function theorem with (4.1) implies that the function $t \mapsto \xi(t)$ is of class $C^{1}\left(\left[\tau_{1},+\infty\right)\right)$. Define also $\xi(t)=\xi\left(\tau_{1}\right)$ for all $t<\tau_{1}$. The function $\xi$ is then continuous in $\mathbb{R}$. We shall show in this Step 4 that (1.13) holds with this function $\xi$. To do so, we first prove in the following two lemmas some key-properties on the local oscillations of the function $\xi$.

Lemma 4.5. There is a positive constant $\tau_{2}$ such that $\xi(t+s)>\xi(t)$ for all $t \geq \tau_{1}$ and $s \geq \tau_{2}$.

Proof. By (1.3) and (4.3), there is a real number $R_{3}>0$ such that

$$
u(t, x)<\varphi(0) \text { for all } t \leq \tau_{1} \text { and } x \text { such that }\left|x-x_{0}\right| \geq R_{3} .
$$

With $\varepsilon=\delta^{2} /\left(4 M_{2}\right)>0, \lambda=(m+M) / 2<M$ and $r=\delta /\left(2 M_{2}\right) \geq 0$, denote, using the notations of Lemma 4.4 and the definition (4.33) of $R_{2}$,

$$
R_{4}=\max \left(\rho_{\varepsilon, \lambda, r}+\frac{\delta}{2 M_{2}}, R_{3}, \xi\left(\tau_{1}\right)+1\right)>0
$$

and

$$
\tau_{2}=T_{\varepsilon, \lambda, r}>0
$$

Let also $\tau_{3} \in \mathbb{R}$ be such that

$$
\xi(t) \geq R_{4} \text { for all } t \geq \tau_{3}
$$

(hence, $\tau_{3}>\tau_{1}$, since $R_{4}>\xi\left(\tau_{1}\right)$ ).

Consider now any $t \geq \tau_{3}$ and $s \geq \tau_{2}$ and let us show that $\xi(t+s)>\xi(t)$. Let $x_{3} \in \mathbb{R}^{N}$ be such that

$$
\left|x_{3}-x_{0}\right|=\xi(t)+R_{1},
$$

where $R_{1}>0$ is given in (4.29). Thus, $\left|x_{3}-x_{0}\right| \geq R_{4}+R_{1} \geq R_{3}+R_{1}$, hence $u\left(t^{\prime}, \cdot\right)<\varphi(0) \leq$ $\varphi\left(\cdot-x_{3}\right)$ in $\overline{B\left(x_{3}, R_{1}\right)}$ for all $t^{\prime} \leq \tau_{1}$ by (4.41). Observe that $\xi\left(t^{\prime}\right)=\xi\left(\tau_{1}\right)<R_{4} \leq \xi(t)$ for all $t^{\prime} \leq \tau_{1}$, and, by continuity of $\xi$, denote

$$
t^{*}=\min \left\{t^{\prime} \in(-\infty, t]: \xi\left(t^{\prime}\right)=\xi(t)\right\} \in\left(\tau_{1}, t\right] .
$$


Let $x_{4}$ be the intersection point of the segment $\left[x_{0}, x_{3}\right]$ with $\partial B\left(x_{3}, R_{1}\right)$. One has

$$
\left|x_{4}-x_{0}\right|=\left|x_{3}-x_{0}\right|-R_{1}=\xi(t)=\xi\left(t^{*}\right),
$$

hence $u\left(t^{*}, x_{4}\right)=m=\varphi\left(x_{4}-x_{3}\right)$. Furthermore, $u\left(t^{\prime}, \cdot\right)<\varphi(0) \leq \varphi\left(\cdot-x_{3}\right)$ in $\overline{B\left(x_{3}, R_{1}\right)}$ for all $t^{\prime} \leq \tau_{1}$ by (4.29) and (4.41), while

$$
u\left(t^{\prime}, \cdot\right) \leq m=\varphi\left(\cdot-x_{3}\right) \text { on } \partial B\left(x_{3}, R_{1}\right) \text { for all } t^{\prime} \in\left[\tau_{1}, t^{*}\right]
$$

by (4.1) and the definition of $t^{*}$ (and even $u\left(t^{\prime}, \cdot\right)<m$ on $\partial B\left(x_{3}, R_{1}\right)$ for all $t^{\prime} \in\left[\tau_{1}, t^{*}\right)$ ). It then follows from the maximum principle that

$$
u\left(t^{\prime}, \cdot\right) \leq \varphi\left(\cdot-x_{3}\right) \text { in } \overline{B\left(x_{3}, R_{1}\right)} \text { for all } t^{\prime} \in\left[\tau_{1}, t^{*}\right]
$$

(actually with strict inequality for $t^{\prime} \in\left[\tau_{1}, t^{*}\right)$ and even for $t^{\prime} \in\left(-\infty, t^{*}\right)$ ). In particular, $u\left(t^{*}, \cdot\right) \leq \varphi\left(\cdot-x_{3}\right)$ in $\overline{B\left(x_{3}, R_{1}\right)}$ and since $x_{4} \in \partial B\left(x_{3}, R_{1}\right)$ with $\left|x_{4}-x_{0}\right|=\xi\left(t^{*}\right)$ and $t^{*} \geq \tau_{1}$, one has $u\left(t^{*}, x_{4}\right)=m=\varphi\left(x_{4}-x_{3}\right)$. Therefore,

$$
-\left|\nabla u\left(t^{*}, x_{4}\right)\right|=\nabla u\left(t^{*}, x_{4}\right) \cdot \frac{x_{4}-x_{0}}{\left|x_{4}-x_{0}\right|} \leq-\delta
$$

owing to the definition of $\delta$ in (4.30)-(4.31). Hence, as in the proof of (4.37), one infers that

$$
u\left(t^{*}, \cdot\right) \geq m+\frac{\delta^{2}}{4 M_{2}}=m+\varepsilon \text { in } \overline{B\left(x_{0},\left|x_{4}-x_{0}\right|-\delta /\left(2 M_{2}\right)\right)},
$$

with $\left|x_{4}-x_{0}\right|-\delta /\left(2 M_{2}\right)=\xi(t)-\delta /\left(2 M_{2}\right) \geq R_{4}-\delta /\left(2 M_{2}\right) \geq \rho_{\varepsilon, \lambda, r}$ by (4.42). Lemma 4.4 then yields

$$
u\left(t^{\prime}, \cdot\right) \geq \lambda=\frac{m+M}{2} \text { in } \overline{B\left(x_{0},\left|x_{4}-x_{0}\right|-\delta /\left(2 M_{2}\right)+r\right)}=\overline{B\left(x_{0},\left|x_{4}-x_{0}\right|\right)}=\overline{B\left(x_{0}, \xi(t)\right)}
$$

for all $t^{\prime} \geq t^{*}+T_{\varepsilon, \lambda, r}=t^{*}+\tau_{2}$. Since $t+s \geq t^{*}+\tau_{2}$, one has $u(t+s, \cdot) \geq(m+M) / 2>m$ in $\overline{B\left(x_{0}, \xi(t)\right)}$ and the definition of $\xi(t+s)$ together with (4.1) and $t+s>t^{*}>\tau_{1}$ finally yields $\xi(t+s)>\xi(t)$.

As a consequence, $\xi(t+s)>\xi(t)$ for all $t \geq \tau_{3}$ and $s \geq \tau_{2}$. Since $\xi$ is continuous in $\mathbb{R}$ and $\xi(t) \rightarrow+\infty$ as $t \rightarrow+\infty$, the conclusion of Lemma 4.5 follows, even if it means increasing $\tau_{2}$ if necessary.

Lemma 4.6. For each $\tau>0$, there is a positive constant $A_{\tau}$ such that $\xi(t+s) \leq \xi(t)+A_{\tau}$ for all $t \in \mathbb{R}$ and $s \in[0, \tau]$.

Proof. Assume that the conclusion does not hold. Then there are $\tau>0$ and some sequences $\left(t_{n}\right)_{n \in \mathbb{N}}$ in $\mathbb{R}$ and $\left(s_{n}\right)_{n \in \mathbb{N}}$ in $[0, \tau]$ such that $\xi\left(t_{n}+s_{n}\right)>\xi\left(t_{n}\right)+n$. Since $\xi$ is continuous in $\mathbb{R}$ and constant in $\left(-\infty, \tau_{1}\right]$, it follows that $t_{n} \rightarrow+\infty$ as $n \rightarrow+\infty$, hence $\xi\left(t_{n}\right) \rightarrow+\infty$ as $n \rightarrow+\infty$ by (4.40). Without loss of generality, one can assume that, for every $n \in \mathbb{N}$,

$$
t_{n} \geq \tau_{1}+\tau_{2} \text { and } \xi\left(t_{n}\right) \geq \max \left(R_{3}, \xi\left(\tau_{1}\right)+1\right)
$$

where $\tau_{1} \in \mathbb{R}$ and $\tau_{2}>0$ are given in (4.39) and in Lemma 4.5, and $R_{3}>0$ is given in (4.41).

Now, for every $n \in \mathbb{N}$, Lemma 4.5 yields the existence of $t_{n}^{*} \in\left(t_{n}-\tau_{2}, t_{n}\right]\left(\subset\left(\tau_{1}, t_{n}\right]\right)$ such that $\xi\left(t_{n}^{*}\right)=\xi\left(t_{n}\right)$ and $\xi(t)<\xi\left(t_{n}^{*}\right)=\xi\left(t_{n}\right)$ for all $t<t_{n}^{*}$. Let $y_{n} \in \mathbb{R}^{N}$ be such that

$$
\left|y_{n}-x_{0}\right|=\xi\left(t_{n}^{*}\right)+R_{1}=\xi\left(t_{n}\right)+R_{1}\left(\geq R_{3}+R_{1}\right) \text {. }
$$


Since $u(t, \cdot)<\varphi(0) \leq \varphi\left(\cdot-y_{n}\right)$ in $\overline{B\left(y_{n}, R_{1}\right)}$ for all $t \leq \tau_{1}$ by (4.41), and since $u(t, \cdot)<$ $m=\varphi\left(\cdot-y_{n}\right)$ on $\partial B\left(y_{n}, R_{1}\right)$ for all $t \in\left[\tau_{1}, t_{n}^{*}\right)$ by (4.1) and definition of $t_{n}^{*}$, the maximum principle implies that

$$
u\left(t_{n}^{*}, \cdot\right) \leq \varphi\left(\cdot-y_{n}\right) \text { in } \overline{B\left(y_{n}, R_{1}\right)} .
$$

In particular, $u\left(t_{n}^{*}, y_{n}\right) \leq \varphi(0)$ and

$$
u\left(t_{n}^{*}, x\right) \leq \varphi(0) \text { for all } x \text { such that }\left|x-x_{0}\right| \geq\left|y_{n}-x_{0}\right|=\xi\left(t_{n}\right)+R_{1},
$$

by (4.1).

On the other hand, for every $n \in \mathbb{N}$, one has $t_{n}+s_{n} \geq t_{n} \geq \tau_{1}+\tau_{2}>\tau_{1}$, and there is a point $z_{n}$ such that $\left|z_{n}-x_{0}\right|=\xi\left(t_{n}+s_{n}\right)$, hence $u\left(t_{n}+s_{n}, z_{n}\right)=m$. Notice also that $t_{n}-t_{n}^{*}+s_{n} \in\left[0, \tau_{2}+\tau\right)$ for each $n \in \mathbb{N}$. Up to extraction of a subsequence, one can assume without loss of generality that $t_{n}-t_{n}^{*}+s_{n} \rightarrow s_{\infty} \in\left[0, \tau_{2}+\tau\right]$ as $n \rightarrow+\infty$ and that the functions

$$
u_{n}:(t, x) \mapsto u_{n}(t, x)=u\left(t+t_{n}^{*}, x+z_{n}\right)
$$

converge in $C_{t, x}^{1,2}$ locally in $\mathbb{R} \times \mathbb{R}^{N}$ to a bounded nonnegative solution $u_{\infty}$ of (1.1) such that $u_{\infty}\left(s_{\infty}, 0\right)=m$. Furthermore, for each $x \in \mathbb{R}^{N}$, there holds

$$
\left|x+z_{n}-x_{0}\right| \geq\left|z_{n}-x_{0}\right|-|x|=\xi\left(t_{n}+s_{n}\right)-|x|>\xi\left(t_{n}\right)+n-|x|,
$$

hence $\left|x+z_{n}-x_{0}\right| \geq \xi\left(t_{n}\right)+R_{1}$ for all $n$ large enough and $u_{n}(0, x)=u\left(t_{n}^{*}, x+z_{n}\right) \leq \varphi(0)$ by (4.45). As a consequence, $u_{\infty}(0, x) \leq \varphi(0)$ for all $x \in \mathbb{R}^{N}$. Since $0<\varphi(0)<\eta$ by (4.29) and $f<0$ in $(0, \eta]$ by (4.8), it follows from the maximum principle that $u_{\infty} \leq \varphi(0)<\eta$ in $[0,+\infty) \times \mathbb{R}^{N}$. In particular, $u_{\infty}\left(s_{\infty}, 0\right)<\eta$, which is impossible since $u_{\infty}\left(s_{\infty}, 0\right)=m$ and $m>\eta$ (remember that $f(m)=0$ and $m>0$ ). One has then reached a contradiction, and the proof of Lemma 4.6 is thereby complete.

With Lemmas 4.5 and 4.6 in hand, we can now complete the proof of (1.13). Let us begin with the first statement in (1.13). Assume by way of contradiction that it does not hold. Then, thanks to (4.11), there are $M^{\prime} \in(0, M)$ and some sequences $\left(t_{n}\right)_{n \in \mathbb{N}}$ converging to $+\infty$ and $\left(x_{n}\right)_{n \in \mathbb{N}}$ in $\mathbb{R}^{N}$ such that

$$
0<u\left(t_{n}, x_{n}\right) \leq M^{\prime}<M \text { for all } n \in \mathbb{N}, \text { and }\left|x_{n}\right|-\xi\left(t_{n}\right) \rightarrow-\infty \text { as } n \rightarrow+\infty .
$$

Let $\tau>0$ be an arbitrary positive real number, and let $R_{1}>0$ be given as in (4.29). Consider in this paragraph the indices $n$ large enough so that $t_{n}-\tau \geq \tau_{1}+\tau_{2}$ for every $n \in \mathbb{N}$, where $\tau_{1} \in \mathbb{R}$ and $\tau_{2}>0$ are given in (4.39) and Lemma 4.5, and

$$
\xi\left(t_{n}-\tau\right) \geq \max \left(R_{3}, \xi\left(\tau_{1}\right)+1, \frac{\delta}{2 M_{2}}+1\right),
$$

where $R_{3}>0$ is given in (4.41), $\delta>0$ in (4.30)-(4.31) and $M_{2}>0$ in (4.32). Notice that the quantities $\tau, \tau_{1}, \tau_{2}, R_{1}, R_{3}, \delta$ and $M_{2}$ are independent of $n$. Now, for each $n$ large enough, Lemma 4.5 yields the existence of $t_{n}^{*} \in\left(t_{n}-\tau-\tau_{2}, t_{n}-\tau\right]\left(\subset\left(\tau_{1}, t_{n}-\tau\right]\right)$ such that $\xi\left(t_{n}^{*}\right)=\xi\left(t_{n}-\tau\right)$ and $\xi(t)<\xi\left(t_{n}^{*}\right)=\xi\left(t_{n}-\tau\right)$ for all $t<t_{n}^{*}$. Let $y_{n} \in \mathbb{R}^{N}$ be such that

$$
\left|y_{n}-x_{0}\right|=\xi\left(t_{n}^{*}\right)+R_{1}=\xi\left(t_{n}-\tau\right)+R_{1}\left(\geq R_{3}+R_{1}\right)
$$


and $z_{n}$ be the intersection point of $\left[x_{0}, y_{n}\right]$ with $\partial B\left(y_{n}, R_{1}\right)$ such that

$$
\left|z_{n}-x_{0}\right|=\left|y_{n}-x_{0}\right|-R_{1}=\xi\left(t_{n}-\tau\right)=\xi\left(t_{n}^{*}\right), \quad u\left(t_{n}^{*}, z_{n}\right)=m
$$

by (4.39). As in the proof of (4.44), there holds $u\left(t_{n}^{*}, \cdot\right) \leq \varphi\left(\cdot-y_{n}\right)$ in $\overline{B\left(y_{n}, R_{1}\right)}$ with $u\left(t_{n}^{*}, z_{n}\right)=m=\varphi\left(z_{n}-y_{n}\right)$. Therefore,

$$
-\left|\nabla u\left(t_{n}^{*}, z_{n}\right)\right|=\nabla u\left(t_{n}^{*}, z_{n}\right) \cdot \frac{z_{n}-x_{0}}{\left|z_{n}-x_{0}\right|} \leq-\delta
$$

with $\delta>0$ given by (4.30)-(4.31). Hence, as in the proof of (4.43), one infers that

$$
u\left(t_{n}^{*}, \cdot\right) \geq m+\frac{\delta^{2}}{4 M_{2}} \text { in } \overline{B\left(x_{0},\left|z_{n}-x_{0}\right|-\delta /\left(2 M_{2}\right)\right)}=\overline{B\left(x_{0}, \xi\left(t_{n}-\tau\right)-\delta /\left(2 M_{2}\right)\right)}
$$

with $\left|z_{n}-x_{0}\right|-\delta /\left(2 M_{2}\right)=\xi\left(t_{n}-\tau\right)-\delta /\left(2 M_{2}\right)>0$. Together with (4.11), this implies in particular that $m<m+\delta^{2} /\left(4 M_{2}\right)<M$. Notice also that $\tau \leq t_{n}-t_{n}^{*}<\tau+\tau_{2}$ for each $n$ (large enough). Up to extraction of a subsequence, one has $t_{n}-t_{n}^{*} \rightarrow s_{\infty} \in\left[\tau, \tau+\tau_{2}\right]$ as $n \rightarrow+\infty$ and the functions

$$
u_{n}:(t, x) \mapsto u_{n}(t, x)=u\left(t+t_{n}^{*}, x+x_{n}\right)
$$

converge in $C_{t, x}^{1,2}$ locally in $\mathbb{R} \times \mathbb{R}^{N}$ to a bounded nonnegative solution $u_{\infty}$ of (1.1) such that

$$
u_{\infty}\left(s_{\infty}, 0\right) \leq M^{\prime}<M
$$

by (4.46). Furthermore, for each $x \in \mathbb{R}^{N}$, one has

$$
\left|x+x_{n}-x_{0}\right| \leq \xi\left(t_{n}\right)+\left|x_{n}\right|-\xi\left(t_{n}\right)+\left|x-x_{0}\right| \leq \xi\left(t_{n}-\tau\right)+A_{\tau}+\left|x_{n}\right|-\xi\left(t_{n}\right)+\left|x-x_{0}\right|
$$

from Lemma 4.6, where $A_{\tau}>0$ is given in Lemma 4.6, hence $\left|x+x_{n}-x_{0}\right| \leq \xi\left(t_{n}-\tau\right)-\delta /\left(2 M_{2}\right)$ for all $n$ large enough, from the second statement of (4.46). As a consequence, $u_{n}(0, x)=$ $u\left(t_{n}^{*}, x+x_{n}\right) \geq m+\delta^{2} /\left(4 M_{2}\right)$ for all $n$ large enough, by (4.47). Thus, $u_{\infty}(0, \cdot) \geq m+\delta^{2} /\left(4 M_{2}\right)$ in $\mathbb{R}^{N}$ and $u_{\infty}(t, \cdot) \geq \varpi(t)$ in $\mathbb{R}^{N}$ for all $t \geq 0$, where $\varpi$ obeys

$$
\left\{\begin{array}{l}
\varpi^{\prime}(t)=f(\varpi(t)) \quad \text { for } t \geq 0, \\
\varpi(0)=m+\frac{\delta^{2}}{4 M_{2}}>m .
\end{array}\right.
$$

Since $m<m+\delta^{2} /\left(4 M_{2}\right)<M$ and $f>0$ in $(m, M)$ with $f(M)=0$, the function $\varpi$ is increasing in $[0,+\infty)$ and $\varpi(+\infty)=M$. The inequality $u_{\infty}(t, \cdot) \geq \varpi(t)$ applied at $t=s_{\infty} \geq$ $\tau>0$ and $x=0$ yields $u_{\infty}\left(s_{\infty}, 0\right) \geq \varpi\left(s_{\infty}\right) \geq \varpi(\tau)$, hence $M>M^{\prime} \geq u_{\infty}\left(s_{\infty}, 0\right) \geq \varpi(\tau)$. Since $M^{\prime}$ is given in (4.46) independently of $\tau>0$ and $\tau>0$ can be arbitrarily large, one infers that $M>M^{\prime} \geq \varpi(+\infty)=M$, a contradiction. As a consequence, the first line in (1.13) has been proved.

Let us now show the second statement in (1.13). Assume by way of contradiction that it does not hold. Then, thanks to (4.11), there are $\kappa \in(0, M)$ and some sequences $\left(t_{n}\right)_{n \in \mathbb{N}}$ converging to $+\infty$ and $\left(x_{n}\right)_{n \in \mathbb{N}}$ in $\mathbb{R}^{N}$ such that

$$
0<\kappa \leq u\left(t_{n}, x_{n}\right)<M \text { for all } n \in \mathbb{N} \text {, and }\left|x_{n}\right|-\xi\left(t_{n}\right) \rightarrow+\infty \text { as } n \rightarrow+\infty .
$$

Let $\sigma>0$ be an arbitrary positive real number such that

$$
\sigma \geq \tau_{2}
$$


where $\tau_{2}>0$ is given in Lemma 4.5, and let $R_{1}>0$ be given as in (4.29). Consider in this paragraph the indices $n$ large enough so that $t_{n}-\sigma \geq \tau_{1}+\tau_{2}$ for every $n \in \mathbb{N}$, where $\tau_{1} \in \mathbb{R}$ is given in (4.39), and

$$
\xi\left(t_{n}-\sigma\right) \geq \max \left(R_{3}, \xi\left(\tau_{1}\right)+1\right),
$$

where $R_{3}>0$ is given in (4.41). For each $n$ large enough, Lemma 4.5 yields the existence of $t_{n}^{*} \in\left(t_{n}-\sigma-\tau_{2}, t_{n}-\sigma\right]\left(\subset\left(\tau_{1}, t_{n}-\sigma\right]\right)$ such that $\xi\left(t_{n}^{*}\right)=\xi\left(t_{n}-\sigma\right)$ and $\xi(t)<\xi\left(t_{n}^{*}\right)=\xi\left(t_{n}-\sigma\right)$ for all $t<t_{n}^{*}$. Let $y_{n} \in \mathbb{R}^{N}$ satisfy

$$
\left|y_{n}-x_{0}\right|=\xi\left(t_{n}^{*}\right)+R_{1}=\xi\left(t_{n}-\sigma\right)+R_{1}\left(\geq R_{3}+R_{1}\right) .
$$

As for (4.44), one then has $u\left(t_{n}^{*}, \cdot\right) \leq \varphi\left(\cdot-y_{n}\right)$ in $\overline{B\left(y_{n}, R_{1}\right)}$. In particular, $u\left(t_{n}^{*}, y_{n}\right) \leq \varphi(0)$ and, from (4.1),

$$
u\left(t_{n}^{*}, x\right) \leq \varphi(0) \text { for all } x \text { such that }\left|x-x_{0}\right| \geq\left|y_{n}-x_{0}\right|=\xi\left(t_{n}-\sigma\right)+R_{1} .
$$

Notice also that $\sigma \leq t_{n}-t_{n}^{*}<\sigma+\tau_{2}$ for each $n$ (large enough). Up to extraction of a subsequence, one has $t_{n}-t_{n}^{*} \rightarrow t_{\infty} \in\left[\sigma, \sigma+\tau_{2}\right]$ as $n \rightarrow+\infty$ and the functions

$$
u_{n}:(t, x) \mapsto u_{n}(t, x)=u\left(t+t_{n}^{*}, x+x_{n}\right)
$$

converge in $C_{t, x}^{1,2}$ locally in $\mathbb{R} \times \mathbb{R}^{N}$ to a bounded nonnegative solution $u_{\infty}$ of (1.1) such that

$$
0<\kappa \leq u_{\infty}\left(t_{\infty}, 0\right)
$$

by (4.48). Furthermore, for each $x \in \mathbb{R}^{N}$, one has

$$
\left|x+x_{n}-x_{0}\right| \geq\left|x_{n}\right|-\xi\left(t_{n}\right)+\xi\left(t_{n}\right)-\left|x-x_{0}\right|>\left|x_{n}\right|-\xi\left(t_{n}\right)+\xi\left(t_{n}-\sigma\right)-\left|x-x_{0}\right|
$$

from Lemma 4.5, since $t_{n}-\sigma \geq \tau_{1}$ and $\sigma \geq \tau_{2}$. Hence $\left|x+x_{n}-x_{0}\right| \geq \xi\left(t_{n}-\sigma\right)+R_{1}$ for all $n$ large enough, from (4.48). As a consequence, $u_{n}(0, x)=u\left(t_{n}^{*}, x+x_{n}\right) \leq \varphi(0)$ for all $n$ large enough, by (4.49). Thus, $u_{\infty}(0, \cdot) \leq \varphi(0)$ in $\mathbb{R}^{N}$ and $u_{\infty}(t, \cdot) \leq \vartheta(t)$ in $\mathbb{R}^{N}$ for all $t \geq 0$, where $\vartheta$ obeys

$$
\left\{\begin{array}{l}
\vartheta^{\prime}(t)=f(\vartheta(t)) \quad \text { for } t \geq 0 \\
\vartheta(0)=\varphi(0) \in(0, \eta) .
\end{array}\right.
$$

Since $f<0$ in $(0, \eta)$ with $f(0)=0$, the function $\vartheta$ is decreasing in $[0,+\infty)$ and $\vartheta(+\infty)=0$. The inequality $u_{\infty}(t, \cdot) \leq \vartheta(t)$ applied at $t=t_{\infty} \geq \sigma>0$ and $x=0$ yields $u_{\infty}\left(t_{\infty}, 0\right) \leq$ $\vartheta\left(t_{\infty}\right) \leq \vartheta(\sigma)$, hence $0<\kappa \leq \vartheta(\sigma)$. Since $\kappa$ is given in (4.48) independently of $\sigma$ and since $\sigma \geq \tau_{2}$ can be arbitrarily large, one infers that $0<\kappa \leq \vartheta(+\infty)=0$, a contradiction. As a conclusion, the proof of (1.13) is thereby complete.

Remark 4.7. The quantities $\xi(t)$ given in (4.39) (for $t \geq \tau_{1}$ ) are the radial positions (with respect to the point $\left.x_{0}\right)$ of the level sets with level $m$. Now, for any level $\lambda$ in $(0, M)$, there is a unique real number $\xi_{\lambda}(t)>0$ such that $u(t, x)=\lambda$ if and only if $\left|x-x_{0}\right|=\xi_{\lambda}(t)$, for all $t$ large enough. One then infers from (1.13) that

$$
\limsup _{t \rightarrow+\infty}\left|\xi_{\lambda}(t)-\xi(t)\right|<+\infty .
$$

Furthermore, it also follows from (4.1) that, for any unit vector $e$ and any sequence $\left(t_{n}\right)_{n \in \mathbb{N}}$ converging to $+\infty$, the functions

$$
u_{n}:(t, x) \mapsto u_{n}(t, x)=u\left(t+t_{n}, x+\xi_{\lambda}\left(t_{n}\right) e\right)
$$


converge in $C_{t, x}^{1,2}$ locally in $\mathbb{R} \times \mathbb{R}^{N}$, up to extraction of a subsequence, to a bounded nonnegative solution $u_{\infty}$ of (1.1) which only depends on $t$ and the variable $x \cdot e$ and is nonincreasing in the direction $e$. Moreover, (1.13) implies that $u_{\infty}(0, x) \rightarrow M$ as $x \cdot e \rightarrow-\infty$ and $u_{\infty}(0, x) \rightarrow 0$ as $x \cdot e \rightarrow+\infty$. In particular, the nonpositive function $e \cdot \nabla u_{\infty}$ can not be identically 0 in $(-\infty, 0] \times \mathbb{R}^{N}$ and the strong parabolic maximum principle then yields $e \cdot \nabla u_{\infty}(0,0)<0$. From the arbitrariness of the sequence $\left(t_{n}\right)_{n \in \mathbb{N}}$ converging to $+\infty$ and from (4.1), we conclude that

$$
\liminf _{t \rightarrow+\infty, u(t, x)=\lambda}|\nabla u(t, x)|>0 \text {, that is, } \limsup _{t \rightarrow+\infty, u(t, x)=\lambda} \nabla u(t, x) \cdot \frac{x-x_{0}}{\left|x-x_{0}\right|}<0,
$$

for any level $\lambda \in(0, M)$. In other words, the radial derivatives of the function $u$ do not degenerate at large times along any level set of $u$.

Step 5: asymptotic position of the level sets if u spreads, proof of (1.14). We still assume that (4.35) holds, hence $M<+\infty$ and (1.13) holds, together with (4.38). We shall show here that $\xi(t) / t$ has a well determined limit as $t \rightarrow+\infty$. Such a property has been well known since the seminal paper [3], under some additional assumptions on the function $f$. The proof given in [3] was based on some comparison arguments and on the existence of approximated fronts defined in bounded intervals or in half-lines. The proof of (1.14) given here is still based on comparison arguments with suitable sub- and supersolutions, but the approximated fronts moving at speeds arbitrarily close to $c$ that are here used are defined in the whole real line and are given in Lemma 3.1 .

First of all, as already emphasized, one has $0<m<M, f(0)=f(M)=0, f^{\prime}(0)<0$, $f>0$ in $(m, M), F<0$ in $(0, m]$ and $F(M)>0$. Lemma 3.1 then yields the existence and uniqueness of a pair $(c, \varphi)$ solving (1.15), with $c>0$.

Consider now any $c^{\prime} \in(0, c)$, and let us show that $\liminf _{t \rightarrow+\infty} \xi(t) / t \geq c^{\prime}$. From Remark 3.2, there is a sequence $\left(\varepsilon_{n}\right)_{n \in \mathbb{N}}$ in $(0, m)$ converging to 0 such that, for each $n \in \mathbb{N}$, there is a $C^{1}\left(\left[-\varepsilon_{n}, M-\varepsilon_{n}\right]\right)$ function $\underline{f}_{n}$ such that $\underline{f}_{n}\left(-\varepsilon_{n}\right)=\underline{f}_{n}\left(M-\varepsilon_{n}\right)=0, \underline{f}_{n} \leq f$ in $\left[0, M-\varepsilon_{n}\right]$, and there is a unique pair $\left(c_{n}, \varphi_{n}\right) \in \mathbb{R} \times C^{2}(\mathbb{R})$ solving

$$
\varphi_{n}^{\prime \prime}+c_{n} \varphi_{n}^{\prime}+\underline{f}_{n}\left(\varphi_{n}\right)=0 \text { in } \mathbb{R}, \varphi_{n}^{\prime}<0 \text { in } \mathbb{R}, \varphi_{n}(-\infty)=M-\varepsilon_{n}, \varphi_{n}(+\infty)=-\varepsilon_{n} .
$$

Furthermore, $c_{n}<c$ and $c_{n} \rightarrow c$ as $n \rightarrow+\infty$. Fix $\varepsilon>0$ arbitrary, and then $n$ large enough such that

$$
0<\varepsilon_{n} \leq \varepsilon \text { and } c^{\prime}<c_{n}<c .
$$

Let then $\rho>0$ large enough such that

$$
\frac{N-1}{\rho}<\frac{c_{n}-c^{\prime}}{2}
$$

and denote $c_{n}^{\prime}=\left(c^{\prime}+c_{n}\right) / 2 \in\left(c^{\prime}, c_{n}\right) \subset\left(c^{\prime}, c\right)$. Since $u(t, \cdot) \rightarrow M$ as $t \rightarrow+\infty$ locally uniformly in $\mathbb{R}^{N}$ by (4.38), there is a time $T>0$ such that

$$
u(t, x) \geq M-\varepsilon_{n} \text { for all } t \geq T \text { and }\left|x-x_{0}\right| \leq \rho .
$$

Let then $A>0$ be such that $\varphi_{n}\left(r-c_{n}^{\prime} T+A\right)<0$ for all $r \geq \rho$ (that is possible since $\left.\varphi_{n}(+\infty)=-\varepsilon_{n}<0\right)$. Let us finally define

$$
\underline{u}(t, x):=\max \left(\varphi_{n}\left(\left|x-x_{0}\right|-c_{n}^{\prime} t+A\right), 0\right)
$$


and show that this function is a generalized subsolution of (1.1) for $t \geq T$ and $\left|x-x_{0}\right| \geq \rho$. First of all, at time $t=T$, for all $\left|x-x_{0}\right| \geq \rho$, one has $\varphi_{n}\left(\left|x-x_{0}\right|-c_{n}^{\prime} T+A\right)<0$, hence $\underline{u}(T, x)=0<u(T, x)$. Furthermore, for all $t \geq T$ and $\left|x-x_{0}\right|=\rho$, one has $\underline{u}(t, x)<M-\varepsilon_{n} \leq u(t, x)$. Since $f(0)=0$, it just remains to show that, for any $(t, x)$ such that $t>T$ and $\left|x-x_{0}\right|>\rho$ with $\underline{u}(t, x)>0$, then $\underline{u}_{t}(t, x) \leq \Delta \underline{u}(t, x)+f(\underline{u}(t, x))$. Pick any such $(t, x)$ and notice that $\underline{u}(t, x)=\varphi_{n}\left(\left|x-x_{0}\right|-c_{n}^{\prime} t+A\right) \in\left(0, M-\varepsilon_{n}\right)$ in a neighborhood of $(t, x)$. Hence, having (4.50) in mind, it follows that

$$
\begin{aligned}
\underline{u}_{t}(t, x)-\Delta \underline{u} & (t, x)-f(\underline{u}(t, x)) \\
= & -c_{n}^{\prime} \varphi_{n}^{\prime}\left(\left|x-x_{0}\right|-c_{n}^{\prime} t+A\right)-\varphi_{n}^{\prime \prime}\left(\left|x-x_{0}\right|-c_{n}^{\prime} t+A\right) \\
& \quad-\frac{N-1}{\left|x-x_{0}\right|} \varphi_{n}^{\prime}\left(\left|x-x_{0}\right|-c_{n}^{\prime} t+A\right)-f\left(\varphi_{n}\left(\left|x-x_{0}\right|-c_{n}^{\prime} t+A\right)\right) \\
\leq & \left(c_{n}-c_{n}^{\prime}-\frac{N-1}{\left|x-x_{0}\right|}\right) \varphi_{n}^{\prime}\left(\left|x-x_{0}\right|-c_{n}^{\prime} t+A\right) \\
& <0
\end{aligned}
$$

since $\underline{f}_{n} \leq f$ in $\left[0, M-\varepsilon_{n}\right],(N-1) /\left|x-x_{0}\right| \leq(N-1) / \rho<\left(c_{n}-c^{\prime}\right) / 2=c_{n}-c_{n}^{\prime}$, and $\varphi_{n}^{\prime}<0$ in $\mathbb{R}$. The maximum principle then implies that

$$
u(t, x) \geq \underline{u}(t, x) \geq \varphi_{n}\left(\left|x-x_{0}\right|-c_{n}^{\prime} t+A\right) \text { for all } t \geq T \text { and }\left|x-x_{0}\right| \geq \rho .
$$

Therefore, together with (4.51), one gets that, for all $t \geq T$,

$$
\min _{\left|x-x_{0}\right| \leq c^{\prime} t} u(t, x) \geq \varphi_{n}\left(c^{\prime} t-c_{n}^{\prime} t+A\right) \rightarrow M-\varepsilon_{n} \text { as } t \rightarrow+\infty,
$$

since $c^{\prime}<c_{n}^{\prime}$ and $\varphi_{n}(-\infty)=M-\varepsilon_{n}$. Together with the inequality $0<u<M$ in $\mathbb{R} \times \mathbb{R}^{N}$, one infers that $\lim \sup _{t \rightarrow+\infty} \max _{\left|x-x_{0}\right| \leq c^{\prime} t}|u(t, x)-M| \leq \varepsilon_{n} \leq \varepsilon$. Since $\varepsilon>0$ and $c^{\prime} \in(0, c)$ were arbitrary, one concludes from the definition (4.39) of $\xi(t)$ that

$$
\liminf _{t \rightarrow+\infty} \frac{\xi(t)}{t} \geq c
$$

For the converse inequality, consider any $c^{\prime \prime}>c$ and let us show that $\lim \sup _{t \rightarrow+\infty} \xi(t) / t \leq c^{\prime \prime}$. From the proof of Lemma 3.1. there is a sequence $\left(\eta_{k}\right)_{k \in \mathbb{N}}$ in $(0, m)$ converging to 0 such that, for each $k \in \mathbb{N}$, there is a $C^{1}\left(\left[\eta_{k}, M+\eta_{k}\right]\right)$ function $\bar{f}_{k}$ such that $\bar{f}_{k}\left(\eta_{k}\right)=\bar{f}_{k}\left(M+\eta_{k}\right)=0$, $\bar{f}_{k} \geq f$ in $\left[\eta_{k}, M\right]$, and there is a unique pair $\left(\gamma_{k}, \phi_{k}\right) \in \mathbb{R} \times C^{2}(\mathbb{R})$ solving

$$
\phi_{k}^{\prime \prime}+\gamma_{k} \phi_{k}^{\prime}+\bar{f}_{k}\left(\phi_{k}\right)=0 \text { in } \mathbb{R}, \phi_{k}^{\prime}<0 \text { in } \mathbb{R}, \phi_{k}(-\infty)=M+\eta_{k}, \phi_{k}(+\infty)=\eta_{k} .
$$

Furthermore, $\gamma_{k}>c$ and $\gamma_{k} \rightarrow c$ as $k \rightarrow+\infty$. Fix $\varepsilon>0$ arbitrary, and then $k$ large enough such that

$$
0<\eta_{k} \leq \varepsilon \text { and } c<\gamma_{k}<c^{\prime \prime}
$$

Since $0<u<M$ in $\mathbb{R} \times \mathbb{R}^{N}$ and $u(0, x) \rightarrow 0$ as $|x| \rightarrow+\infty$ by (1.3), and since $\phi_{k}(-\infty)=$ $M+\eta_{k}>M$ and $\phi_{k}>\phi_{k}(+\infty)=\eta_{k}>0$, there is $A^{\prime}>0$ such that $\phi_{k}\left(1-A^{\prime}\right)>M$ and $u(0, x)<\phi_{k}\left(\left|x-x_{0}\right|-A^{\prime}\right)$ for all $x \in \mathbb{R}^{N}$. Let us finally define

$$
\bar{u}(t, x):=\min \left(\phi_{k}\left(\left|x-x_{0}\right|-\gamma_{k} t-A^{\prime}\right), M\right)
$$

and show that this function is a generalized supersolution of (1.1) for $t \geq 0$ and $\left|x-x_{0}\right| \geq 1$. First of all, at time $t=0$, one has $u(0, x)<\bar{u}(0, x)$ for all $\left|x-x_{0}\right| \geq 1$ (and even for 
all $x \in \mathbb{R}^{N}$, by construction). Furthermore, for all $t \geq 0$ and $\left|x-x_{0}\right|=1$, one has $\gamma_{k} t \geq 0$ and $\phi_{k}\left(\left|x-x_{0}\right|-\gamma_{k} t-A^{\prime}\right) \geq \phi_{k}\left(1-A^{\prime}\right)>M$, hence $\bar{u}(t, x)=M>u(t, x)$. Since $f(M)=0$, it just remains to show that, for any $(t, x)$ such that $t>0$ and $\left|x-x_{0}\right|>1$ with $\bar{u}(t, x)<M$, then $\bar{u}_{t}(t, x) \geq \Delta \bar{u}(t, x)+f(\bar{u}(t, x))$. Pick any such $(t, x)$ and notice that $\bar{u}(t, x)=\phi_{k}\left(\left|x-x_{0}\right|-\gamma_{k} t-A^{\prime}\right) \in\left(\eta_{k}, M\right)$ in a neighborhood of $(t, x)$. Hence, having (4.53) in mind, it follows that

$$
\begin{aligned}
\bar{u}_{t}(t, x)-\Delta \bar{u} & (t, x)-f(\bar{u}(t, x)) \\
= & -\gamma_{k} \phi_{k}^{\prime}\left(\left|x-x_{0}\right|-\gamma_{k} t-A^{\prime}\right)-\phi_{k}^{\prime \prime}\left(\left|x-x_{0}\right|-\gamma_{k} t-A^{\prime}\right) \\
& \quad-\frac{N-1}{\left|x-x_{0}\right|} \phi_{k}^{\prime}\left(\left|x-x_{0}\right|-\gamma_{k} t-A^{\prime}\right)-f\left(\phi_{k}\left(\left|x-x_{0}\right|-\gamma_{k} t-A^{\prime}\right)\right) \\
& \geq-\frac{N-1}{\left|x-x_{0}\right|} \phi_{k}^{\prime}\left(\left|x-x_{0}\right|-\gamma_{k} t-A^{\prime}\right) \\
& \geq 0
\end{aligned}
$$

since $\bar{f}_{k} \geq f$ in $\left[\eta_{k}, M\right]$ and $\phi_{k}^{\prime}<0$ in $\mathbb{R}$. The maximum principle then implies that

$$
u(t, x) \leq \bar{u}(t, x) \leq \phi_{k}\left(\left|x-x_{0}\right|-\gamma_{k} t-A^{\prime}\right) \text { for all } t \geq 0 \text { and }\left|x-x_{0}\right| \geq 1 .
$$

Therefore, for all $t \geq 1 / c^{\prime \prime}$, one has $\max _{\left|x-x_{0}\right| \geq c^{\prime \prime} t} u(t, x) \leq \phi_{k}\left(c^{\prime \prime} t-\gamma_{k} t-A^{\prime}\right) \rightarrow \eta_{k}$ as $t \rightarrow+\infty$, since $c^{\prime \prime}>\gamma_{k}$ and $\phi_{k}(+\infty)=\eta_{k}$. One then infers that

$$
\limsup _{t \rightarrow+\infty} \max _{\left|x-x_{0}\right| \geq c^{\prime \prime} t} u(t, x) \leq \eta_{k} \leq \varepsilon .
$$

Since $\varepsilon>0$ and $c^{\prime \prime}>c$ were arbitrary, one concludes from the definition (4.39) of $\xi(t)$ that $\lim \sup _{t \rightarrow+\infty} \xi(t) / t \leq c$. Together with (4.52), the inequality (1.14) follows. The proof of Theorem 1.1 is thereby complete.

\section{Proof of Corollaries 2.1 and 2.3}

As already emphasized in Section 2, Corollary 2.2 follows directly from Theorem 1.1, while Corollaries 2.4 2.7 follow from Corollaries 2.2 and 2.3 and the results of Section 1. It just remains to complete the proof of Corollaries 2.1 and 2.3 .

Proof of Corollary 2.1. From the observations in the paragraph before Corollary 2.1 and from the assumptions made in Corollary 2.1, there is a unique solution $\phi_{0}$ of (1.4) such that $\max _{\mathbb{R}} \phi_{0}=\phi_{0}(0)=\beta$ (and $\phi_{0}$ is then even and decreasing in $|x|$ ). Furthermore, $0<m_{\phi_{0}}<\beta$ and $F<0$ in $\left(0, m_{\phi_{0}}\right]$. All conditions of Theorem 1.1 are then fulfilled.

Let now $u$ be a positive bounded solution of (1.1) satisfying (1.3). As in (4.1), there is $x_{0} \in \mathbb{R}$ such that, for every $t \in \mathbb{R}$, the function $x \mapsto u\left(t, x+x_{0}\right)$ is even in $x$ and decreasing in $|x|$. Since $\phi:=\phi_{0}\left(\cdot-x_{0}\right)$ is the only solution of (1.4) which is symmetric with respect to $x_{0}$, it follows from property (1.12) of Theorem 1.1 that

$$
\|u(t, \cdot)-\phi\|_{L^{\infty}(\mathbb{R})} \rightarrow 0 \text { as } t \rightarrow-\infty .
$$

Lastly, if alternative (ii) holds in the conclusion of Theorem 1.1, then again by the uniqueness of the symmetric (with respect to $x_{0}$ ) solution $\phi$ of (1.4), one has $\|u(t, \cdot)-\phi\|_{L^{\infty}(\mathbb{R})} \rightarrow 0$ as $t \rightarrow+\infty$. It then follows from (4.7), (4.9) and (4.36) that the action $E[u(t, \cdot)]$ defined 
by (4.5) has the same limit $E[\phi]$ as $t \rightarrow \pm \infty$. From (4.6), one concludes that $u_{t} \equiv 0$ in $\mathbb{R} \times \mathbb{R}$, that is, $u(t, x) \equiv \phi(x)$ in $\mathbb{R} \times \mathbb{R}$. The proof of Corollary 2.1 is thereby complete.

Proof of Corollary 2.3. As in (4.1), there is a point $x_{0} \in \mathbb{R}^{N}$ such that, for every $t \in \mathbb{R}$, the function $x \mapsto u\left(t, x+x_{0}\right)$ is radially symmetric, and decreasing in $|x|$. From the assumptions made in Corollary 2.3, the set of solutions of (1.4) which are radially symmetric with respect to the point $x_{0}$ is discrete. Since the $\alpha$-limit set of $u$ is non-empty, connected and made of solutions of (1.4) which are radially symmetric with respect to $x_{0}$ (from the proof of (1.12) in Section 4.1), it follows that there is $\phi \in \mathcal{E}$ such that

$$
\|u(t, \cdot)-\phi\|_{L^{\infty}\left(\mathbb{R}^{N}\right)} \rightarrow 0 \text { as } t \rightarrow-\infty .
$$

In case alternative (ii) of the conclusion of Theorem 1.1 occurs, then there is $\phi^{\prime} \in \mathcal{E}$ such that $\left\|u(t, \cdot)-\phi^{\prime}\right\|_{L^{\infty}\left(\mathbb{R}^{N}\right)} \rightarrow 0$ as $t \rightarrow+\infty$. Furthermore, if $\phi=\phi^{\prime}$, then, as in the above proof of Corollary 2.1, one has $E[u(t, \cdot)] \rightarrow E[\phi]=E\left[\phi^{\prime}\right]$ as $t \rightarrow \pm \infty$, hence $u_{t} \equiv 0$ and $u(t, x) \equiv \phi(x) \equiv \phi^{\prime}(x)$ in $\mathbb{R} \times \mathbb{R}^{N}$.

\section{ACKNOWLEDGEMENTS}

This work has been carried out in the framework of Archimède Labex of Aix-Marseille University. The project leading to this publication has received funding from Excellence Initiative of Aix-Marseille University - A*MIDEX, a French "Investissements d'Avenir" programme, and from the ANR NONLOCAL project (ANR-14-CE25-0013). The second author was partially supported by JSPS KAKENHI Grant Numbers JP16KT0022 and JP20H01816 and also thanks Aix-Marseille University for the warm hospitality during the visit. The authors are grateful to Thomas Giletti, Louis Jeanjean, Peter Poláčik, and Luca Rossi for helpful discussions and useful references.

\section{REFERENCES}

[1] S. Adachi, M. Shibata, and T. Watanabe, A note on the uniqueness and the non-degeneracy of positive radial solutions for semilinear elliptic problems and its application, Acta Math. Sci. B 38 (2018), 11211142 .

[2] S. Allen and J. W. Cahn, A microscopic theory for antiphase boundary motion and its application to antiphase domain coarsening, Acta. Metall. 27 (1979), 1084-1095.

[3] D. G. Aronson and H. F. Weinberger, Multidimensional nonlinear diffusion arising in population genetics, Adv. Math. 30 (1978), 33-76.

[4] H. Berestycki, Th. Gallouët, and O. Kavian, Équations de champs scalaires euclidiens non linéaires dans le plan, C. R. Acad. Sci. Paris Sér. I Math. 297 (1983), 307-310.

[5] H. Berestycki and F. Hamel, Generalized transition waves and their properties, Comm. Pure Appl. Math. 65 (2012), 592-648.

[6] H. Berestycki and P.-L. Lions, Une méthode locale pour l'existence de solutions positives de problèmes semilinéaires elliptiques dans $\mathbb{R}^{N}$, J. Anal. Math. 38 (1980), 144-187.

[7] H. Berestycki and P.-L. Lions, Nonlinear scalar fields, I: Existence of a ground state, Arch. Ration. Mech. Anal. 82 (1983), 313-345.

[8] H. Berestycki, P.-L. Lions, and L. A. Peletier, An ODE approach to the existence of positive solutions for semilinear problems in $\mathbb{R}^{n}$, Indiana Univ. Math. J. 30 (1981), 141-157.

[9] J. Busca, M. A. Jendoubi, and P. Poláčik, Convergence to equilibrium for semilinear parabolic problems in $\mathbb{R}^{N}$, Comm. Part. Diff. Equations 27 (2002), 1793-1814. 
[10] C.-C. Chen and C.-S. Lin, Uniqueness of the ground state solution of $\Delta u+f(u)=0$ in $\mathbb{R}^{n}, n \geq 3$, Comm. Part. Diff. Equations 16 (1991), 1549-1572.

[11] C. V. Coffman, Uniqueness of the ground state solution for $\Delta u-u+u^{3}=0$ and a variational characterization of other solutions, Arch. Ration. Mech. Anal. 46 (1972), 81-95.

[12] C. Cortazar, M. Elgueta, and P. Felmer, Uniqueness of positive solution of $\Delta u+f(u)=0$ in $\mathbb{R}^{N}, N \geq 3$, Arch. Ration. Mech. Anal. 142 (1998), 127-141.

[13] J. Dávila, M. del Pino, and I. Guerra, Non-uniqueness of positive ground states of non-linear Schrödinger equations, Proc. London Math. Soc. 106 (2013), 318-344.

[14] Y. Du and H. Matano, Convergence and sharp thresholds for propagation in nonlinear diffusion problems, J. Europ. Math. Soc. 12 (2010), 279-312.

[15] Y. Du and H. Matano, Radial terrace solutions and propagation profile of multistable reaction-diffusion equations over $\mathbb{R}^{N}$, https://arxiv.org/pdf/1711.00952.pdf.

[16] Y. Du and P. Poláčik, Locally uniform convergence to an equilibrium for nonlinear parabolic equations on $\mathbb{R}^{N}$, Indiana Univ. Math. J. 64 (2015), 787-824.

[17] A. Ducrot, T. Giletti, and H. Matano, Existence and convergence to a propagating terrace in onedimensional reaction-diffusion equations, Trans. Amer. Math. Soc. 366 (2014), 5541-5566.

[18] J.-P. Eckmann and J. Rougemont, Coarsening by Ginzburg-Landau dynamics, Comm. Math. Phys. 199 (1998), 441-470.

[19] E. Feireisl, On the long time behavior of solutions to nonlinear diffusion equations on $\mathbb{R}^{N}$, Nonlinear Diff. Eq. Appl. 4 (1997), 43-60.

[20] P. Fife and J. B. McLeod, The approach of solutions of nonlinear diffusion equations to travelling front solutions, Arch. Ration. Mech. Anal. 65 (1977), 335-361.

[21] M. Fila and E. Yanagida, Homoclinic and heteroclinic orbits for a semilinear parabolic equation, Tohoku Math. J. 63 (2011), 561-579.

[22] J. Földes and P. Poláčik, Convergence to a steady state for asymptotically autonomous semilinear heat equations on $\mathbb{R}^{N}$, J. Diff. Equations 251 (2011), 1903-1922.

[23] B. Franchi, E. Lanconelli, and J. Serrin, Existence and uniqueness of nonnegative solutions of quasilinear equations in $\mathbb{R}^{n}$, Adv. Math. 118 (1996), 177-243.

[24] Y. Fukao, Y. Morita, and H. Ninomiya, Some entire solutions of Allen-Cahn equation, Taiwanese J. Math. 8 (2004), 15-32.

[25] Th. Gallay and S. Slijepčević, Distribution of energy and convergence to equilibria in extended dissipative systems, J. Dyn. Diff. Equations 27 (2015), 653-682.

[26] B. Gidas, W.-M. Ni, and L. Nirenberg, Symmetry and related properties via the maximum principle, Comm. Math. Phys. 68 (1979), 209-243.

[27] B. Gidas, W.-M. Ni, and L. Nirenberg, Symmetry of positive solutions of nonlinear elliptic equations in $\mathbb{R}^{n}$, Adv. Math. Suppl. Studies A 7 (1981), 369-402.

[28] T. Giletti and H. Matano, Existence and uniqueness of propagating terraces, Commun. Contemp. Math., forthcoming.

[29] T. Giletti and L. Rossi, Pulsating solutions for multidimensional bistable and multistable equations, Math. Ann., forthcoming.

[30] J.-S. Guo and Y. Morita, Entire solutions of reaction-diffusion equations and an application to discrete diffusive equations, Disc. Cont. Dyn. Syst. 12 (2005), 193-212.

[31] J.-S. Guo, H. Ninomiya, M. Shimojo and E. Yanagida, Convergence and blow-up of solutions for a complex-valued heat equation with quadratic nonlinearity, Trans. Amer. Math. Soc. 365 (2013), 24472467.

[32] J. K. Hale and G. Raugel, Convergence in gradient-like systems with applications to PDE, Z. angew. Math. Phys. 43 (1992), 63-124.

[33] F. Hamel and N. Nadirashvili, Travelling fronts and entire solutions of the Fisher-KPP equation in $\mathbb{R}^{N}$, Arch. Ration. Mech. Anal. 157 (2001), 91-163.

[34] F. Hamel and J.-M. Roquejoffre, Heteroclinic connections for multidimensional bistable reactiondiffusion equations, Disc. Cont. Dyn. Syst. S 4 (2011), 101-123. 
[35] C. K. R. T. Jones, Spherically symmetric solutions of a reaction-diffusion equation, J. Diff. Equations 49 (1983), 142-169.

[36] I. Kuzin and S. I. Pohožaev, Entire Solutions of Semilinear Elliptic Equations, Progress Nonlin. Diff. Eq. Appl. 33, Birkhaüser, 1997.

[37] M. K. Kwong, Uniqueness of positive solution of $\Delta u-u+u^{p}=0$ in $\mathbb{R}^{n}$, Arch. Ration. Mech. Anal. 105 (1989), 243-266.

[38] C. Li, Monotonicity and symmetry of solutions of fully nonlinear elliptic equations on unbounded domains, Comm. Part. Diff. Equations 16 (1991), 585-615.

[39] Y. Li and W.-N. Ni, Radial symmetry of positive solutions of nonlinear elliptic equations in $\mathbb{R}^{n}$, Comm. Part. Diff. Equations 18 (1993), 1043-1054.

[40] H. Matano, Existence of nontrivial unstable sets for equilibriums of strongly order-preserving systems J. Fac. Sci. Univ. Tokyo Sect. IA Math. 30 (1984), 645-673.

[41] H. Matano and P. Poláčik, An entire solution of a bistable parabolic equation on $\mathbb{R}$ with two colliding pulses, J. Funct. Anal. 272 (2017), 1956-1979.

[42] H. Matano and P. Poláčik, Dynamics of nonnegative solutions of one-dimensional reaction-diffusion equations with localized initial data. Part I: A general quasiconvergence theorem and its consequences, Comm. Part. Diff. Equations 41 (2016), 785-811.

[43] H. Matano and P. Poláčik, Dynamics of nonnegative solutions of one-dimensional reaction-diffusion equations with localized initial data. Part II: Generic nonlinearities, Comm. Part. Diff. Equations, forthcoming.

[44] K. McLeod, Uniqueness of positive radial solutions of $\Delta u+f(u)=0$ in $\mathbb{R}^{n}$, II, Trans. Amer. Math. Soc. 339 (1993), 495-505.

[45] K. McLeod and J. Serrin, Uniqueness of positive radial solutions of $\Delta u+f(u)=0$ in $\mathbb{R}^{n}$, Arch. Ration. Mech. Anal. 99 (1987), 115-145.

[46] Y. Morita and H. Ninomiya, Monostable-type traveling waves of bistable reaction-diffusion equations in the multi-dimensional space, Bull. Inst. Math. Acad. Sin. 3 (2008), 567-584.

[47] C. B. Muratov and X. Zhong, Threshold phenomena for symmetric decreasing solutions of reactiondiffusion equations, Nonlin. Diff. Eq. Appl. 20 (2013), 1519-1552.

[48] C. B. Muratov and X. Zhong, Threshold phenomena for symmetric-decreasing radial solutions of reaction-diffusion equations, Disc. Cont. Dyn. Syst. 37 (2017), 915-944.

[49] J. Nagumo, S. Yoshizawa, and S. Arimoto, Bistable transmission lines, IEEE Trans. Circuit Theory 12 (1965), 400-412.

[50] W.-M. Ni and J. Serrin, Nonexistence theorems for quasilinear partial differential equations, Rend. Circolo Mat. Palermo 8 (1985), 171-185.

[51] L. A. Peletier and J. Serrin, Uniqueness of positive solutions of semilinear equations in $\mathbb{R}^{n}$, Arch. Ration. Mech. Anal. 81 (1983), 181-197.

[52] S. I. Pohožaev, On the eigenfunctions of the equation $\Delta u+\lambda f(u)=0$, Dokl. Akad. Nauk SSSR 165 (1965), 36-39.

[53] P. Poláčik, Morse indices and bifurcations of positive solutions of $\Delta u+f(u)=0$ on $\mathbb{R}^{N}$, Indiana Univ. Math. J. 50 (2001), 1407-1432.

[54] P. Poláčik, Symmetry properties of positive solutions of parabolic equations on $\mathbb{R}^{N}$, II: Entire solutions, Comm. Part. Diff. Equations 31 (2006), 1615-1638.

[55] P. Poláčik, Examples of bounded solutions with nonstationary limit profiles for semilinear heat equations on $\mathbb{R}$, J. Evol. Equations 15 (2015), 281-307.

[56] P. Poláčik, Threshold behavior and non-quasiconvergent solutions with localized initial data for bistable reaction-diffusion equations, J. Dyn. Diff. Equations 28 (2016), 605-625.

[57] P. Poláčik, Convergence and quasiconvergence properties of solutions of parabolic equations on the real line: an overview, In: Patterns of Dynamics, Springer Proc. Math. Stat. 205, Springer, Cham 2017, $172-183$.

[58] P. Poláčik, Propagating terraces in a proof of the Gibbons conjecture and related results, J. Fixed Point Theory Appl. 19 (2017), 113-128. 
[59] P. Poláčik, Planar propagating terraces and the asymptotic one-dimensional symmetry of solutions of semilinear parabolic equations, SIAM J. Math. Anal. 49 (2017), 3716-3740.

[60] P. Poláčik and E. Yanagida, On bounded and unbounded global solutions of a supercritical semilinear heat equation, Math. Ann. 327 (2003), 745-771.

[61] P. Poláčik and E. Yanagida, Localized solutions of a semilinear parabolic equation with a recurrent nonstationary asymptotics, SIAM J. Math. Anal. 46 (2014), 3481-3496.

[62] E. Risler, Global behaviour of bistable solutions for gradient systems in one unbounded spatial dimension, https://arxiv.org/pdf/1604.02002.pdf.

[63] E. Risler, Global behaviour of radially symmetric solutions stable at infinity for gradient systems, https://arxiv.org/pdf/1703.02134.pdf.

[64] L. Rossi, The Freidlin-Gärtner formula for general reaction terms, Adv. Math. 317 (2017), 267-298.

[65] V. Roussier, Stability of radially symmetric travelling waves in reaction-diffusion equations, Ann. Inst. H. Poincaré, Anal. Non Linéaire 21 (2004), 341-379.

[66] J. Serrin and M. Tang, Uniqueness of ground states for quasilinear elliptic equations, Indiana Univ. Math. J. 49 (2000), 897-923.

[67] W. A. Strauss, Existence of solitary waves in higher dimensions, Comm. Math. Phys. 55 (1977), $149-162$.

[68] K. Uchiyama, Asymptotic behavior of solutions of reaction-diffusion equations with varying drift coefficients, Arch. Ration. Mech. Anal. 90 (1985), 291-311.

[69] E. Yanagida, Uniqueness of positive radial solution of $\Delta u+g(r) u+h(r) u^{p}=0$ in $\mathbb{R}^{n}$, Arch. Ration. Mech. Anal. 115 (1991), 257-274.

(F. Hamel) Aix Marseille Univ, CNRS, Centrale Marseille, I2M, Marseille, France E-mail address: francois.hamel@univ-amu.fr

(H. Ninomiya) School of Interdisciplinary Mathematical Sciences, Meiji University 4-21-1 NAKANO, NAKANO-KU, TOKYO 164-8525, JAPAN

E-mail address: hirokazu.ninomiya@gmail.com 